\title{
No evidence for a special role of language in feature-based categorization
}

\author{
Yael Benn ${ }^{*}$, Anna A. Ivanova ${ }^{2,3}$, Oliver Clark ${ }^{1}$, Zachary Mineroff ${ }^{2,3}$, Chloe Seikus ${ }^{4}$, Jack $^{2}$ \\ Santos Silva ${ }^{4}$, Rosemary Varley ${ }^{\dagger 4}$, Evelina Fedorenko ${ }^{\dagger 2,3}$ \\ ${ }^{1}$ Department of Psychology, Manchester Metropolitan University, Manchester, UK \\ ${ }^{2}$ Brain and Cognitive Sciences Department, Massachusetts Institute of Technology, \\ Cambridge, MA, USA \\ ${ }^{3}$ McGovern Institute for Brain Research, Massachusetts Institute of Technology, Cambridge, \\ MA, USA \\ ${ }^{4}$ Division of Psychology \& Language Sciences, University College London, London, UK \\ * Co-first authors \\ ${ }^{\dagger}$ Co-senior authors
}

For submission to: Cognition

This research did not receive any specific grant from funding agencies in the public, commercial, or not-for-profit sectors. We would like to acknowledge the Athinoula A. Martinos Imaging Center at the McGovern Institute for Brain Research at MIT, and its support team (Steve Shannon and Atsushi Takahashi). EF was supported by NIH awards R00-HD057522, R01-DC016607, R01-DC016950, a grant from the Simons Foundation to the Simons Center for the Social Brain at MIT, and by support from the Brain and Cognitive Sciences Department and the McGovern Institute for Brain Research at MIT. The authors thank Naveen Hanif and Anis Adila Khairil Anuar, who have helped with development and piloting of the paradigms used in Studies 1 and 2, and Alvincé Pongos for help with data analysis.

Address correspondence to: Yael Benn, Department of Psychology, Manchester Metropolitan University, Brooks Building, Birley Fields Campus, 53 Bonsall Street, Manchester, M15 6GX. Email: y.benn@mmu.ac.uk 


\begin{abstract}
The role of language in mediating or augmenting human thought is the subject of longstanding debate. One specific claim links language and the ability to categorize objects based on a certain feature. According to this view, language resources are critical for feature-based categorization because verbal labels can help maintain focus on the relevant categorization criterion and reduce interference from other (irrelevant) features. As a result, language impairment is expected to affect categorization of items grouped according to a single feature (low-dimensional categories, e.g., 'Things made of wood'), where many irrelevant features need to be inhibited, more than categorization of items that share many features (highdimensional categories, e.g., 'Animals'), where few irrelevant features need to be inhibited. We here present findings from individuals with aphasia that go against this hypothesis (Experiments 1 and 2). We also present fMRI data from young healthy adults, showing that the language brain regions exhibit low activity level during categorization, for both lowdimensional and high-dimensional categories (Experiment 3). In tandem, these results demonstrate that language is not critical for object categorization. Our work contributes to the growing evidence that, although language may assist in accessing task-relevant information (e.g., instructions), many cognitive tasks in adult brains proceed without recruiting the language system.
\end{abstract}

Word count: 205

Keywords: Categorization, Language, Aphasia, fMRI 


\section{Introduction}

The role of language in mediating or augmenting thought is the subject of long-standing debate. According to one view, language is necessary for many cognitive functions, such as math, logic, and (inner) thought (e.g., Baldo et al., 2010, 2015; Bermúdez, 2007; Bickerton, 1995; Carruthers, 2002; Darwin, 1871; Dennett, 1994). However, a large body of evidence supports a different view: that language is cognitively and neurally independent from the rest of human cognition. This evidence includes the lack of activity in the language brain regions during non-linguistic tasks that allegedly require language (e.g., Amalric \& Dehaene, 2016, 2019; Fedorenko et al., 2011; Ivanova et al., 2021; Monti et al., 2009, 2012), the retained ability of some individuals with aphasia to perform such tasks (e.g., Bek et al., 2013; Benn et al., 2013; Siegal \& Varley, 2006; Varley et al., 2005), and variability across cultures in the use of language resources during thought (Kim, 2002). However, the role of language is still contested for one important aspect of human cognition: categorization.

Like other animals, humans can convert rich, multi-dimensional perceptual inputs into a latent lower-dimensional structured representation of the world. Grouping discriminable individual objects and events into object and event classes allows us not only to decide whether some new object/event belongs to a particular category, but also to draw powerful inferences about shared properties from one category member to another (e.g., Mareschal \& Quinn, 2001; Mervis \& Rosch, 1981; Murphy, 2002; Pearce, 1994; E. E. Smith \& Medin, 1981; L. B. Smith \& Heise, 1992; Wasserman et al., 1988). In contrast to other animals, humans additionally label individual categories with words - the core building blocks of a powerful communication system that allows us to share complex thoughts with one another. The link between words and learning the structure of new categories has been extensively investigated in infants/children (e.g., Ferguson \& Waxman, 2017; Gershkoff-Stowe et al., 1997; Plunkett et al., 2008; Sloutsky \& Fisher, 2004; Waxman \& Gelman, 2009) and, to some extent, in adults (Brojde et al., 2011; Lupyan et al., 2007; Lupyan \& Casasanto, 2015). But how does language affect the process of grouping objects into categories when the category boundaries are already known?

\subsection{High-dimensional and low-dimensional categories}

Before summarizing the key evidence, it is important to introduce a distinction that has been considered theoretically and empirically relevant to this question. Lupyan and colleagues 
(e.g., Lupyan, 2009; Lupyan \& Mirman, 2013; Perry \& Lupyan, 2014) distinguish between 'high-dimensional' (HD) categories, where members share many features, and 'lowdimensional' (LD) categories, where members share one or a few features. HD categories typically correspond to established sets that reflect either the taxonomic (similarity-based) or relational/thematic (contiguity- or co-occurrence-based) structure of the world (Bain, 1864; Mirman et al., 2017). Taxonomic HD categories can often be labeled by superordinate terms such as ANIMALS, FRUIT, or TOOLS. Relational HD categories correspond to common events/scenarios: for example, THINGS YOU TAKE ON A PICNIC or NON-FOOD THINGS FOUND IN THE KITCHEN. For such relational categories, the shared features have to do with typical co-occurrences (e.g., although a fridge and a spatula are quite different, they both co-occur with a large number of kitchen objects, like a stove, pots and pans, a kettle, etc.). In contrast to HD categories, LD categories are more likely to be novel groupings of items that often straddle taxonomic and relational boundaries, such as THINGS MADE OF WOOD or THINGS THAT ARE YELLOW (e.g., things made of wood may include a cupboard, a sledge, and a wooden spoon, and things that are yellow may include a lemon, a yellow hat, and a canary).

Similar distinctions have been made by others in related literatures. For example, Barsalou (1983) distinguishes between 'common' categories, which mirror the correlational structure of the environment, and 'ad-hoc', or 'goal-derived', categories, which are constructed for a specific goal and are thus often based on a small number of features. Kloos \& Sloutsky (2008) and Sloutsky (2010) distinguish between 'dense' and 'sparse' categories based on the ratio of category-relevant variance to total variance. Members of statistically dense categories share many inter-correlated features that matter for category membership with few or no irrelevant features, and members of sparse categories have very few features in common, with many other features varying independently and being irrelevant for category membership. Couchman et al. (2010) contrast family-resemblance categorization, which relies on judgments of overall similarity, considering multiple features in tandem, and criterial-attribute categorization (or 'rule-based categorization'), which requires adhering to a single-dimensional criterial attribute and suppressing all other, irrelevant dimensions (see also Ashby \& O'Brien, 2005). Langland-Hassan et al. (2021) relate the HD/LD distinction to the concrete/abstract distinction, arguing that concrete items have many shared features, whereas identifying items from an abstract category requires generalizing over many irrelevant properties to identify a small set of commonalities. 


\subsection{The LD-specific language recruitment hypothesis}

One claim that emerged in the literature in recent years is that language plays a special role in LD categorization (Lupyan, 2009, 2012; Lupyan \& Mirman, 2013). The argument goes as follows: categorizing objects into $\mathrm{LD} /$ sparse categories is more cognitively costly because features irrelevant to the categorization criterion interfere and have to be inhibited (e.g., when categorizing objects by color, their shape and function have to be ignored); a verbal label (e.g., 'yellow') can help maintain focus on the relevant categorization criterion ${ }^{1}$ and reduce interference from irrelevant features. The LD-specific language recruitment hypothesis makes two predictions: i) reduced availability of language resources should lead to a greater disruption of $\mathrm{LD}$ compared to $\mathrm{HD}$ categorization (we will adhere to the 'LD'/'HD' terminology in the remainder of the paper for consistency with Lupyan and colleagues' work); and ii) LD categorization should engage the language system to a greater degree than HD categorization.

The first prediction had found some support in the aphasia literature. Some patients with linguistic deficits have been reported to exhibit impairments in non-verbal categorization tasks when the task required focusing on one particular dimension and ignoring other salient dimensions (Cohen et al., 1980; Cohen \& Woll, 1981; Davidoff \& Roberson, 2004; De Renzi \& Spinnler, 1967; Hjelmquist, 1989). Building on these findings, Lupyan (2009) manipulated verbal vs. spatial interference in a dual-task paradigm in neurotypical participants and found that verbal, but not visuo-spatial, interference affected the participants' ability to decide whether an object belongs to an LD category. In contrast, verbal and visuo-spatial interference had similar (and negligible) effects on HD categorization. Lupyan and colleagues concluded that access to lexical resources (verbal labels) is important for LD categorization.

In a follow-up study, Lupyan and Mirman (2013) directly compared performance on HD and LD categorization in individuals with aphasia and neurotypical controls. Participants were provided with a category label and then had to select from a picture array the subset of objects that belong to the target category (similar to Figure 1, top). Performance in the LD

\footnotetext{
${ }^{1}$ In some places, Lupyan and colleagues talk about 'forming the task-relevant category representations' (e.g., Lupyan \& Mirman, 2013, p. 1188). However, this construal is confusing given that in the paradigms used in these studies, the category label is always provided to the participants. As a result, we assume that the argument has to revolve around the maintenance of task-relevant information.
} 
condition was lower for both groups, but critically, the HD vs. LD difference was larger in individuals with aphasia, particularly in those with low scores on a picture-naming task.

However, evidence from aphasia does not provide uniform support for the LD-specific language recruitment hypothesis. For example, Burger and Muma (1980) found deficits in HD categorization in individuals with anomia and in individuals with Wernicke's aphasia using a task similar to that used in Lupyan and Mirman (2013). Others described aphasiarelated categorization deficits for both HD and LD categories (Koemeda-Lutz et al., 1987) or no deficits in either (Hough, 1993). Further, variations in the task (such as showing the category label to the participant during the entire trial vs. just at the beginning of the trial) significantly affected categorization performance in participants with aphasia (Koemeda-Lutz et al., 1987), suggesting that task demands may contribute to the observed results (above and beyond alleged effects of category type). Finally, some have argued for a relationship between categorization difficulties and conceptual-semantic rather than linguistic impairments (Caramazza et al., 1982; Whitehouse et al., 1978; cf. Le Dorze \& Nespoulous, 1989).

It is important to emphasize that even if individuals with aphasia did consistently show a selective impairment in LD categorization, it would not necessarily implicate language as the source of the deficit. In particular, the language network in the left hemisphere, especially in the left frontal cortex, lies adjacent to the domain-general multiple demand network, which supports executive functions, like working memory and inhibitory control (Assem, Glasser, et al., 2020; Duncan, 2010, 2013; Fedorenko et al., 2012, 2013). As a result, left hemisphere damage can lead to joint linguistic and domain-general executive deficits (Baldo et al., 2010; Gainotti et al., 1986). Prior work has shown that performance on executive function tasks, not language tasks, predicts success in learning novel categories (Vallila-Rohter \& Kiran, 2015). Further, the multiple demand network, but not the language network, is robustly sensitive to cognitive effort across domains (e.g., Fedorenko et al., 2011, 2013; Hugdahl et al., 2015; Shashidhara et al., 2019), and LD categorization appears to be more cognitively challenging than HD categorization: LD categories are harder to learn for both human children (e.g., Kloos \& Sloutsky, 2008) and non-human primates (Couchman et al., 2010), require supervision (e.g., Kloos \& Sloutsky, 2008), and are generally linked with executively-taxing intentional learning (Ashby et al., 1998; Ashby \& Ell, 2001; Ashby \& O’Brien, 2005; Couchman et al., 2010; Kemler Nelson, 1984). Finally, information about LD category 
membership is typically not stored but rather "computed on the fly", which can also result in higher cognitive load. It is therefore possible that impaired performance on LD categorization (and on categorization tasks more broadly) depends primarily on domain-general executive resources.

The second prediction of the LD-specific language recruitment hypothesis is that LD categories would evoke stronger activity within the language brain regions. To our knowledge, this hypothesis has not been directly tested in the neuroimaging literature; instead, many studies have investigated differences between taxonomic and thematic relations (e.g., Kalénine et al., 2009; Lewis et al., 2015; Sachs et al., 2008; Sass et al., 2009), both of which are considered HD. Further, few neuroimaging studies employ methods that would be required to dissociate the contributions of language-specific regions from those of domain-general executive regions: given the inter-individual variability in the precise locations of functional areas, voxels in anatomically identical locations within the frontal lobe might be language-specific in one individual and domain-general in another, so traditional group-based analyses (Friston et al., 1994) would fail to distinguish between them (Fedorenko et al., 2012; Fedorenko \& Blank, 2020; Nieto-Castañón \& Fedorenko, 2012). Unambiguously assessing the role of language in $\mathrm{LD}$ categorization requires identification of language-specific regions in individual participants and testing their responses to LD compared to HD conditions.

\subsection{Current study}

Here, we report three interlinked experiments aimed at re-examining the role of language in categorization. In line with recent emphasis on robust and replicable science (e.g., Ioannidis, 2014; Poldrack et al., 2017), in Experiments 1 and 2, we attempt to conceptually replicate the findings of Lupyan and Mirman (2013; L\&M henceforth). In Experiment 1, we closely follow L\&M's experimental procedure, but additionally include another brain-damaged control group (individuals with Parkinson's disease, or PD) to examine general effects of brain damage on performance. In Experiment 2, we adjust the experimental paradigm to reduce general executive demands, which might affect performance (e.g., Koemeda-Lutz et al., 1987). Finally, in Experiment 3, we use fMRI in neurotypical individuals to test the prediction that the language system is engaged during LD categorization more than during HD categorization. 
To foreshadow our results, we find that participants with aphasia perform worse on the categorization task overall, but this effect does not selectively affect LD categories. In Experiment 1, participants with aphasia actually performed better on LD trials than on HD trials. In Experiment 2, participants with low naming scores did show impaired performance on $\mathrm{LD}$ categorization compared to HD categorization; however, the performance of one individual with severe naming impairments was within 2 standard deviations of healthy controls on both LD and HD categorization. Finally, Experiment 3 revealed low engagement of the language network during both LD and HD categorization, with no significant difference between the two. Thus, the language system does not play a special role in LD (single-feature-based) categorization and is not engaged during categorization in general.

\section{Experiment 1}

The aim of Experiment 1 was to replicate the effects reported by L\&M. In their study, L\&M compared LD and HD categorization performance in participants with anomic aphasia and in neurotypical controls. They found a) lower performance on LD compared to HD categories in both healthy adults and participants with anomic aphasia; and, critically, b) a greater decrement in performance for the $\mathrm{LD}$, compared to the HD condition in participants with aphasia. We aimed to see whether these same effects would be present in our study. To additionally examine the extent to which performance might depend on the general effect of brain damage, as opposed to a linguistic impairment, we also included a group of individuals with Parkinson's disease (PD).

\subsection{Methods}

\subsubsection{Participants}

Neurotypical older participants $(N=9$ (6 F), age $M=67.89, S D=14.98)$ were recruited by convenience sampling; individuals with chronic aphasia ( $N=11$ (3 F), age $M=61.18$, $S D=12.09$ ) were recruited from the UCL Aphasia Clinic Research Register. The aphasia group included patients with a range of aphasia types and severities. Individuals with PD ( $N=14(8 \mathrm{~F})$, age $M=68.64, S D=11.69)$ were recruited from the Parkinson's UK Research Registry (see Table 1 for detailed participant information). All participants used English as their primary language. Patients were offered a $£ 10.00$ reimbursement. Ethical approval was 

available under aCC-BY 4.0 International license.

granted by the UCL Research Ethics panel, Project ID: LC/2013/05, and all volunteers gave informed consent to participate in the experiment. 
Table 1. Participant information, Experiment 1.

\begin{tabular}{|c|c|c|c|c|c|c|}
\hline Group & Participant & Age & Education & Gender & $\begin{array}{c}\text { TPO } \\
\text { (months) }\end{array}$ & BNT \\
\hline \multirow[t]{9}{*}{ Neurotypical } & 1 & 75 & Up to 16 & $\mathrm{~F}$ & - & 51 \\
\hline & 2 & 68 & Up to 16 & $\mathrm{~F}$ & - & 55 \\
\hline & 3 & 68 & Up to 16 & M & - & 55 \\
\hline & 4 & 56 & Degree-Level & $\mathrm{F}$ & - & 59 \\
\hline & 5 & 98 & Up to 16 & $\mathrm{~F}$ & - & 47 \\
\hline & 6 & 54 & Degree-Level & $\mathrm{M}$ & - & 53 \\
\hline & 7 & 69 & Up to 16 & M & - & 55 \\
\hline & 8 & 76 & Up to 16 & $\mathrm{~F}$ & - & 52 \\
\hline & 9 & 47 & Up to 18 & $\mathrm{~F}$ & - & 58 \\
\hline \multirow[t]{14}{*}{ PD } & 1 & 60 & Postgraduate & M & 36 & 59 \\
\hline & 2 & 58 & Degree-Level & M & 12 & 58 \\
\hline & 3 & 80 & Up to 18 & $\mathrm{~F}$ & 48 & 58 \\
\hline & 4 & 56 & Postgraduate & $\mathrm{F}$ & 48 & 54 \\
\hline & 5 & 66 & Degree-Level & $\mathrm{F}$ & 72 & 59 \\
\hline & 6 & 75 & Degree-Level & $\mathrm{F}$ & 96 & 56 \\
\hline & 7 & 76 & Up to 18 & M & 36 & 54 \\
\hline & 8 & 59 & Degree-Level & $\mathrm{F}$ & 60 & 55 \\
\hline & 9 & 69 & Postgraduate & $\mathrm{F}$ & 36 & 54 \\
\hline & 10 & 63 & Postgraduate & $\mathrm{F}$ & 60 & 56 \\
\hline & 11 & 77 & Degree-Level & $\mathrm{M}$ & 12 & 46 \\
\hline & 12 & 72 & Postgraduate & M & 120 & 53 \\
\hline & 13 & 75 & Degree-Level & M & 2 & 58 \\
\hline & 14 & 75 & Postgraduate & $\mathrm{F}$ & 360 & 53 \\
\hline \multirow[t]{11}{*}{ Aphasia } & 1 & 52 & Degree-Level & M & 120 & 30 \\
\hline & 2 & 57 & Up to 16 & M & 84 & 57 \\
\hline & 3 & 52 & Up to 18 & M & 48 & 52 \\
\hline & 4 & 59 & Postgraduate & M & 120 & 43 \\
\hline & 5 & 79 & Up to 16 & $\mathrm{~F}$ & 36 & 50 \\
\hline & 6 & 44 & Up to 18 & $\mathrm{~F}$ & 12 & 14 \\
\hline & 7 & 81 & Up to 16 & M & 96 & 57 \\
\hline & 8 & 56 & Up to 18 & M & 60 & 12 \\
\hline & 9 & 57 & Up to 18 & M & 48 & 51 \\
\hline & 10 & 60 & Up to 16 & M & 132 & 34 \\
\hline & 11 & 76 & Up to 16 & $\mathrm{~F}$ & 84 & 14 \\
\hline
\end{tabular}

TPO- Time Post Onset; BNT- Boston Naming Test

\subsubsection{Design and Materials}

The critical categorization task was modeled closely on L\&M's experiment, which used 34 unique categories (18 HD categories and $16 \mathrm{LD}$ categories), with some repetition of categories in each condition. We chose to not repeat any categories, so we limited the materials to 16 categories in each condition (dropping 'BODY PARTS' and 'FACIAL FEATURES' from the HD set). Unlike L\&M, who used cartoon images, we used highquality color photographs selected from the Hemera Photo Objects 5000 and Google Images. For each category, we selected 8-15 targets and 25-27 distractors, for a total of 1087 unique images (any given image appeared as a target in 0-2 categories and as a distractor in 0-2 categories). All photographs depicted objects on a white background. The materials and the experimental scripts for all experiments are available on OSF: https://osf.io/guwh8/. 
To determine the extent of lexical impairment in the aphasia group and to compare lexical abilities across the three groups, all participants completed the Boston Naming Test (BNT; Goodglass et al., 1983), where they were sequentially presented with up to 60 line drawings of objects and asked to overtly name each one. The standard discontinuation rule was applied, with testing stopped after eight consecutive failed naming attempts. No semantic or phonological cues were given.
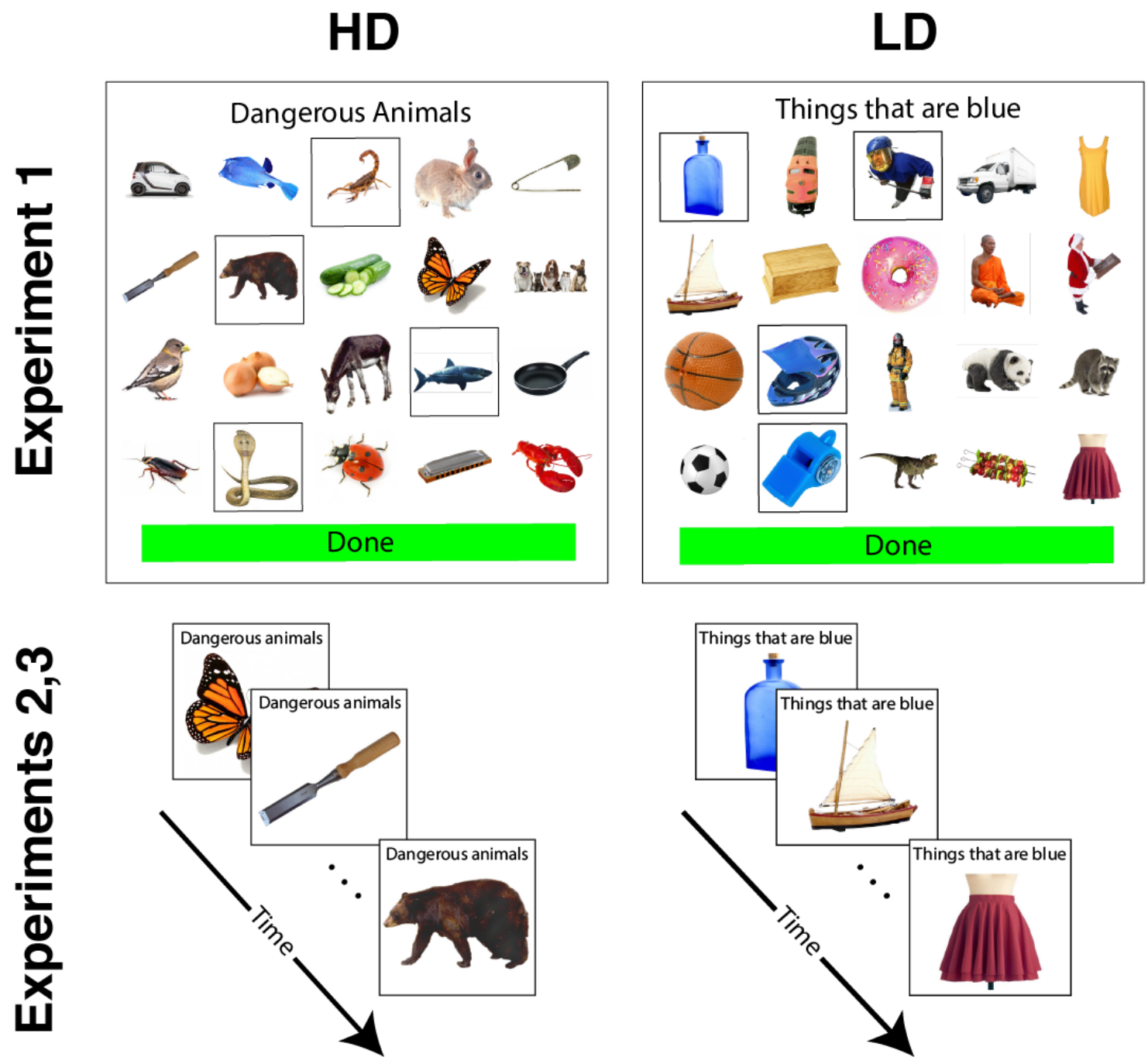

Figure 1. Trial structure in Experiment 1 (top) and Experiments 2 and 3 (bottom). HD - high dimensional category, $L D$ - low dimensional category.

\subsubsection{Experimental Procedure}

Testing was carried out individually either in a quiet well-lit room at the UCL Aphasia clinic or at the participants' home, using a MacBook Pro (Retina, 13-inch display) and an external 
computer mouse. The experiment was set up using PsychoPy (Version 1.83), and the procedure closely followed that used in L\&M's experiment, except where noted. On each trial (see Figure 1 (top) for a sample HD and LD trial), participants were presented with a 4 x 5 grid of images. The image sets for the individual trials-each consisting of 20 images (4 targets and 16 distractors)-were randomly selected from the pool of targets/distractors for each participant separately. The category was stated at the top of the screen in lower-case Arial bold letters (e.g., 'objects that hold water') and remained on the screen for the duration of the trial. Participants selected the objects that belonged to the target category by clicking on each relevant image. A gray frame appeared around an image once it was clicked; clicking the image again de-selected it (removed the gray frame) to allow participants to modify responses. Once the participant had selected all of the images they deemed appropriate for the target category, they clicked a large green button with the word 'Done' at the bottom of the screen (in the L\&M version, the button said 'click here when done'). Doing so triggered the next trial. Although each trial contained a fixed number of targets (four), participants were not informed of the number of targets during the instructions and could therefore select as many images as they wished on any given trial. No time limit was imposed on the trials, but participants were encouraged to work as quickly and accurately as possible. HD and LD trials were interleaved, and the order of conditions was randomized for each participant. Each participant performed the experiment twice for a total of 64 trials (32 per condition), but in contrast to L\&M, different sets of images were used for the two instances of each category to minimize practice effects. Responses were recorded for each image; response times were recorded for each trial (the time from the onset of the trial until the 'Done' button was pressed $^{2}$ ). The experiment lasted approximately one hour. The BNT (Goodglass et al., 2001) was administered between the two runs of the experiment.

\subsubsection{Statistical analyses}

To determine possible differences in demographics and BNT scores across groups, we conducted ANOVA tests (with follow-up Bonferroni-corrected t-tests), implemented in SPSS 22 (IBM Corp., 2013). For the critical analyses, we used linear/logistic mixed effect

\footnotetext{
${ }^{2}$ L\&M state that they only included 'the correct responses' in their RT analyses. It is not clear what is meant here given the internal complexity of the trials (i.e., possible errors including misses and false alarms). It is possible that L\&M only included trials where no errors of any kind were made, but they also talk about 'per click' RTs, which is not consistent with this interpretation. It also appears that L\&M analyzed median, not mean RTs. For simplicity and to avoid collider bias (Elwert \& Winship, 2014), we chose to analyze all trials here. We use mean per-trial values, but we make the per-image data available on OSF (https://osf.io/guwh8/), so other researchers could perform additional analyses.
} 
regression models (Baayen et al., 2008). Given that correct or incorrect selection of items is categorical in nature, we use logistic regression to analyze accuracy measures (Jaeger, 2008). For response times, we use linear regression. When specifying model contrasts, we used sum coding for category dimension (HD vs. LD); the effect of group was therefore estimated across both category dimensions. For participant group (neurotypical vs. aphasia vs. PD), we used dummy coding with 'neurotypical' as the reference level; thus, the effect of category was estimated specifically for the neurotypical group (with interaction terms denoting whether the category effect differed for the aphasia/PD groups). For completeness and to facilitate result comparison with $\mathrm{L} \& \mathrm{M}$, we also ran pairwise comparisons across groups using 'aphasia' as the reference level (the results were Bonferroni-corrected, $n=2$ ). The mixed effect analyses were run using the lmer function from the lme4 $\mathrm{R}$ package (D. Bates et al., 2015); statistical significance of the effects was evaluated using the lmerTest package (Kuznetsova et al., 2017); follow-up comparisons were conducted using the emmeans package (https://cran.r-project.org/package=emmeans). Lastly, due to a technical error, if participants accidently double-clicked the 'Done' button, the next set of images was skipped, and the software registered it as though no response was made by participants. As a result, we excluded trials where no selection was made and where the trial length was less than 5 seconds. This resulted in the exclusion of 42 trials (out of 2177), spread randomly between participants, groups and categories. The analysis code is available on OSF: https://osf.io/guwh8/.

\subsection{Results}

\subsubsection{Group profiles}

As expected, the neurotypical, aphasia, and PD groups differed significantly in their BNT scores $(F(2,31)=9.85, p<.001)$. Post-hoc pairwise comparisons showed that the BNT scores of participants with aphasia $(M=37.64, S D=17.78)$ were significantly lower than those of neurotypical participants $(p=.005)$ or participants with PD $(p=.001)$, with the latter two groups not differing significantly $(M=53.89, S D=3.66$ vs. $M=55.21, S D=3.42 ; p>.999)$. The groups did not differ in age $(F(2,31)=1.45, p=.250)$, but a significant difference was observed in the level of education $(F(2,31)=14.36, p<.001)$ : participants with PD group were significantly more educated than both neurotypical participants $(p=.001)$ and participants with aphasia ( $p=.002)$, with the latter two not differing significantly $(p>.999)$. 


\subsubsection{Categorization task}

Following L\&M, three dependent variables were analyzed: hit rate (the number of targets selected out of 4), false alarm rate (the number of distractors selected out of 16), and trial response time (RT). To account for heterogeneity among participants and experimental categories, we used mixed effect regression models (Baayen et al., 2008) with category dimension (HD vs. LD), group (neurotypical, aphasia, and PD) and their interaction as fixed effects, as well as category (e.g. "DANGEROUS ANIMALS") and participant ID as random effect intercepts. Categorization results are summarized in Figure 2.
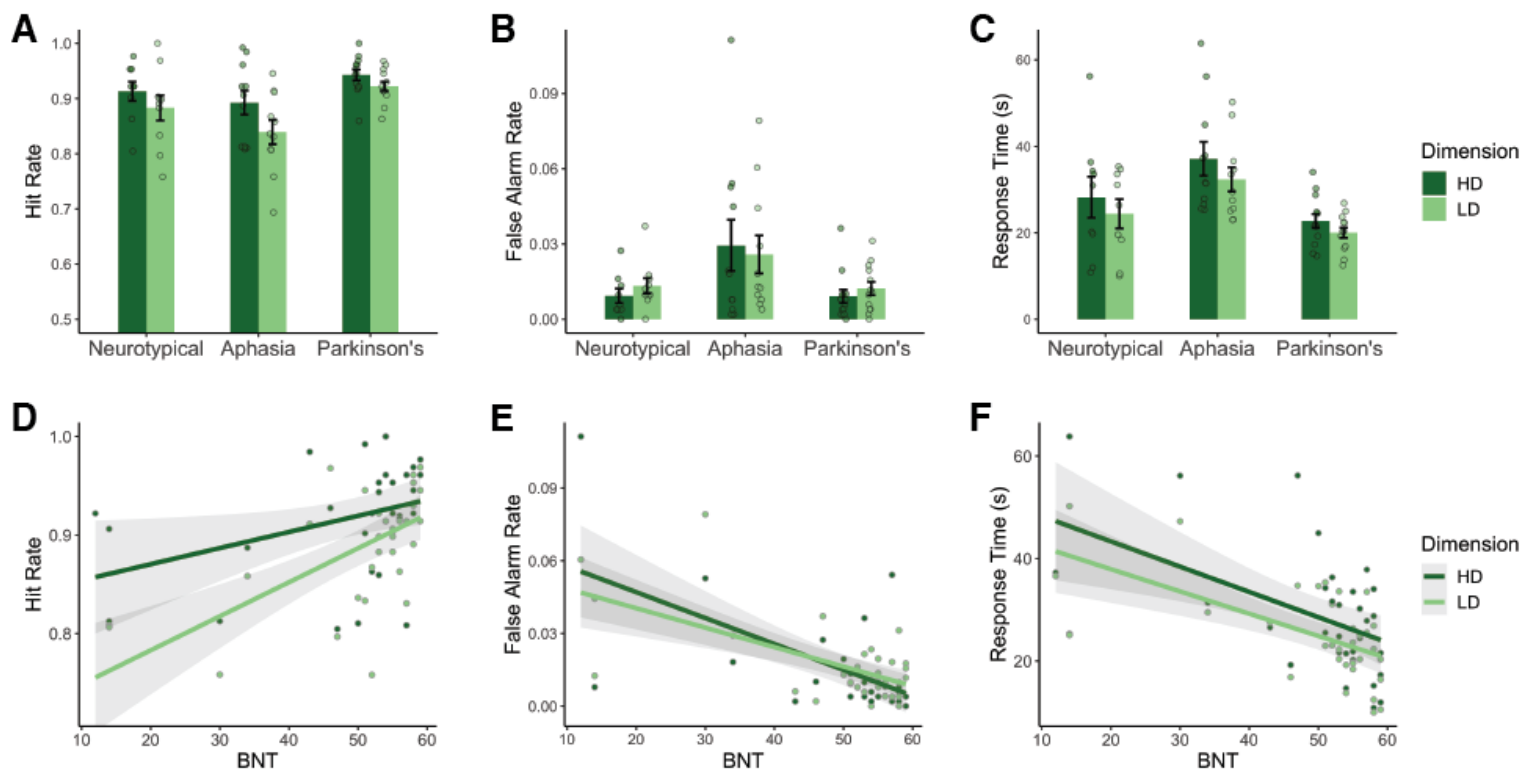

Figure 2. Experiment 1 results. (A) Hit Rate, (B) False Alarm Rate, and (C) Response Time (RT) across the three participant groups (here, $R T$ is the time from trial onset until participants pressed the “Done” button). (D) Hit Rate, (E) False Alarm rate, and (F) RT plotted against participants' BNT scores, a measure of naming performance. Here and elsewhere, error bars depict the standard error across participants.

Hit rate. Participants with aphasia had similar hit rates for $\mathrm{LD}$ categories $(M=0.84, S D=0.07)$ and $\mathrm{HD}$ categories $(M=0.89, S D=0.07$; $\mathrm{LD}>\mathrm{HD}: \beta=-0.41, S E=0.28, p=.139)$. The overall hit rate for participants with aphasia $(M=0.87, S D=0.08)$ was similar to neurotypical participants $(M=0.90, S D=0.06$; neurotypical $>$ aphasia: $\beta=0.24, S E=0.25, p=.338)$ and lower than for participants with $\mathrm{PD}(M=0.93, S D=0.03$; $\mathrm{PD}>$ aphasia: $\beta=0.72, S E=0.23, p=.002)$. Moreover, we did not observe a reliable category dimension by group interaction for the aphasia vs. 
neurotypical comparison $(\beta=0.04, S E=0.18, p=.813)$, nor for the aphasia vs. PD comparison ( $\beta=0.19, S E=0.18, p=.304)$. Follow-up analyses showed that there was no main effect of category dimension across groups $(\beta=0.34, S E=0.27, p=.813)$, nor within the neurotypical group $(\beta=0.37, S E=0.29, p=.478)$ or the PD group $(\beta=0.22, S E=0.29, p=.788)$. These results fail to replicate the findings by L\&M, who reported the main effect of category dimension, as well as a selective impairment in LD categorization for patients with aphasia (Table 2).

Table 2. Statistical significance ( $p$ values) of group and category dimension effects on categorization performance. The critical result reported by $L \& M$ is the Aphasia/Neurotypical (NT) Group by LD/HD interaction - highlighted in yellow - that we do not replicate. Here and below, $p$ values in the L\&M paper were obtained with ANOVA tests, whereas $p$ values in our experiments were obtained with linear/logistic mixed effect models. See the main text for analysis details.

\begin{tabular}{|c|c|c|c|c|}
\hline Experiment & Factor & Hit Rate & False Alarm & Reaction Time \\
\hline \multirow[t]{3}{*}{ Lupyan \& Mirman } & Dimension (across all groups) & $<.001$ & $<.001$ & n.s. ${ }^{\dagger}$ \\
\hline & Group: NT vs. aphasia & .086 & n.s. & .001 \\
\hline & $\begin{array}{l}\text { Group (NT vs. aphasia) } \\
\text { x Dimension }\end{array}$ & .032 & .160 & .038 \\
\hline \multirow[t]{5}{*}{ Ours - Experiment 1} & Dimension (across all groups) & .813 & .951 & .249 \\
\hline & Group: NT vs. aphasia & .338 & .123 & .044 \\
\hline & $\begin{array}{l}\text { Group (NT vs. aphasia) } \\
\text { x Dimension }\end{array}$ & .813 & $.0066^{\dagger \dagger}$ & .522 \\
\hline & $\begin{array}{l}\text { Group - PD vs. aphasia } \\
\text { Group (PD vs. aphasia) }\end{array}$ & .002 & .031 & $<.001$ \\
\hline & x Dimension & .304 & .034 & .091 \\
\hline \multirow[t]{5}{*}{ Ours - Experiment 2} & Dimension (across all groups) & .990 & .985 & .339 \\
\hline & $\begin{array}{l}\text { Group: NT vs. aphasia } \\
\text { Groun (NT vs aphasia) }\end{array}$ & $<.001$ & $<.001$ & $<.001$ \\
\hline & $\mathrm{x}$ Dimension & .086 & .075 & $<.001$ \\
\hline & $\begin{array}{l}\text { Group: PD vs. aphasia } \\
\text { Group (PD vs. aphasia) }\end{array}$ & .005 & $<.001$ & $<.001$ \\
\hline & x Dimension & .070 & .381 & $<.001$ \\
\hline
\end{tabular}

We additionally conducted an exploratory analysis to investigate the difference between the aphasia and PD groups. Given that the PD group had a higher average education level, we repeated the analysis above with 'education level' as an additional fixed effect. The updated model had a similar fit to the data compared to the original (as per the likelihood ratio test: $\chi^{2}=3.39, p=.065$ ); under this model, the difference between the aphasia and the PD groups 
was no longer significant $(\beta=0.37, S E=0.29, p=.202)$. The significance of other effects was unchanged.

False alarm rate. The false alarm rate in participants with aphasia also did not differ between LD categories $(M=0.03, S D=0.03)$ and HD categories $(M=0.03, S D=0.03$; LD $>$ HD: $\beta=-0.22$, $S E=0.35, p=.534)$. As with the hit rate, the overall false alarm rate for participants with aphasia $(M=0.03, S D=0.03)$ was comparable to that of neurotypical participants $(M=0.01$, $S D=0.01$; neurotypical >aphasia: $\beta=-0.58, S E=0.37, p=.123)$, although participants with PD performed better than participants with aphasia, i.e., with fewer false alarms $(M=0.01$, $S D=0.01$; PD>aphasia: $\beta=-0.74, S E=.34, p=.031)$. Unlike the hit rate results above, there was a significant interaction between category dimension (LD>HD) and group (neurotypical $>$ aphasia: $\beta=0.63, S E=0.23, p=.006$; $\mathrm{PD}>$ aphasia: $\beta=0.44, S E=0.21, p=.034$ ). However, this interaction effect goes in the opposite direction from that predicted by the LDspecific language recruitment hypothesis: participants with aphasia performed better on LD categories relative to controls. The pattern of results is also inconsistent with L\&M's results in that they found no interaction between group and category dimension. Lastly, follow-up analyses showed no main effect of category dimension across groups $(\beta=-0.14, S E=0.34$, $p=.951)$, nor within the neurotypical group $(\beta=-0.41, S E=0.38, p=.614)$ or the PD group $(\beta=-$ $0.22, S E=0.37, p=.879)$.

Similar to the hit rate analysis, an exploratory model that included 'education level' as a fixed effect explained a similar amount of variance compared to the original model $\left(\chi^{2}=0.35\right.$, $p=.557)$ and no longer showed a significant difference between the aphasia and PD groups ( $\beta=-0.57, S E=0.45, p=.209)$. The significance of other effects was unchanged.

Response time. The RT analysis revealed that participants with aphasia were faster to respond during LD trials $(M=32.36, S D=9.33)$ compared to HD trials $(M=37.10, S D=12.90$; LD>HD: $\beta=-4.75, S E=2.26, p=.042$ ), in contrast to the predictions of the LD-specific language recruitment hypothesis. The overall RTs for participants with aphasia $(M=34.70$, $S D=11.30)$ were longer than for neurotypical participants $(M=26.30, S D=12.10 ; \beta=-8.42$, $S E=4.02, p=.044)$ and the PD group $(M=21.40, S D=5.14 ; \beta=-13.30, S E=3.66, p<.001)$. The interactions between group and category dimension were not significant (neurotypical >aphasia: $\beta=0.82, S E=1.29, p=.522$; PD>aphasia: $\beta=1.98, S E=1.17, p=.091$ ). Follow-up analyses showed no overall effect of category dimension across groups $(\beta=3.81$, 
$S E=2.19, p=.249)$, within the neurotypical group $(\beta=3.92, S E=2.34, p=.271)$ or within the PD group $(\beta=2.76, S E=2.27, p=.521)$.

Effect of naming performance. To explore the effect of naming ability on the categorization task performance, we fitted a logistic mixed effect linear regression model with the BNT score, category dimension, and their interaction as fixed effects and participants (across the three groups) and categories (e.g., "DANGEROUS ANIMALS") as random effects. Similar to L\&M, we also included education level as a fixed effect.

Table 3. Statistical significance ( $p$ values) of category dimension and anomia effects on categorization performance. The critical result reported by $L \& M$ is the Aphasia/Neurotypical Group by LD/HD interaction - highlighted in yellow - that we replicate in Experiment 2 but not in Experiment 1. The effect of naming performance on response times is not reported in $L \& M$, so we omit it from this table.

\begin{tabular}{|c|c|c|c|}
\hline Experiment & Factor & Hit Rate & False Alarm \\
\hline \multirow[t]{3}{*}{ Lupyan \& Mirman } & Dimension & $?^{\dagger}$ & $?^{\dagger}$ \\
\hline & PNT & .015 & n.s. \\
\hline & PNT x Dimension & .015 & n.s. \\
\hline \multirow[t]{3}{*}{ Ours - Experiment 1} & Dimension & .236 & .690 \\
\hline & $\mathrm{BNT}$ & $<.001$ & $<.001$ \\
\hline & BNT x Dimension & .078 & $.011^{\dagger \dagger}$ \\
\hline \multirow[t]{3}{*}{ Ours - Experiment 2} & Dimension & .885 & .639 \\
\hline & $\mathrm{BNT}$ & .002 & $<.001$ \\
\hline & BNT x Dimension & .039 & .090 \\
\hline
\end{tabular}

We found that BNT was a significant predictor of hit rate $(\beta=0.28, S E=0.08, p<.001)$, false alarm rate $(\beta=-0.47, S E=0.12, p<.001)$, and $\mathrm{RT}(\beta=-5.26, S E=1.41, p<.001)$, such that higher BNT scores corresponded to more accurate and faster performance (Figure 2D-F). There was no main effect of category dimension (hit rate: $\beta=-0.31, S E=0.27, p=.236$; false alarm rate: $\beta=0.14, S E=0.35, p=.690 ; \mathrm{RT}: \beta=-3.73, S E=2.14, p=.092)$. However, we observed an interaction between BNT and category dimension for false alarm rate $(\beta=0.16, S E=0.16$, $p=.011$ ), such that participants with lower BNT scores had higher false alarm rates for HD compared to LD categories. No interaction was observed for group and category dimension 
for hit rate $(\beta=0.11, S E=0.07, p=.078)$ or $\mathrm{RT}(\beta=0.74, S E=0.49, p=.131)$. Conversely, education was a significant predictor for hit rate $(\beta=0.23, S E=0.07, p=.001)$ and $\mathrm{RT}(\beta=-2.95$, $S E=1.24, p=.024)$, but not for false alarm rate $(\beta=-0.09, S E=0.11, p=.436)$. These results do not replicate the pattern of results reported by L\&M (Table 3) and do not support the LDspecific language recruitment hypothesis.

\subsection{Interim Discussion}

In Experiment 1, we aimed to test the hypothesis that language is selectively recruited to support LD categorization by using a setup similar to L\&M's study. Our results differed from those reported in the original study and did not support the LD-specific language recruitment hypothesis. None of the three outcome measures (hit rate, false alarm rate, RT) differed between LD and HD categorization conditions. Moreover, we did not replicate the key finding from L\&M: participants with aphasia showed no selective impairment in LD categorization. The interactions between group and category dimension for hit rate and RT were not significant, and the interaction for the false alarm rate went in the opposite direction from that predicted by the original hypothesis. In summary, Experiment 1 provides no support for the hypothesis that language plays a special role in LD categorization.

With regard to group differences, participants with aphasia performed as accurately as the neurotypical controls. Participants with PD performed better, but this difference is likely explained by the higher education level reported by participants in this group. As in L\&M, our findings showed that participants with aphasia were significantly slower to complete the categorization task compared to the neurotypical and PD groups. However, the reason for this slower performance can be explained by the presence of more severe motor impairments in participants with aphasia than participants with PD (e.g., right hemiplegia), often necessitating use of their non-preferred hand. Thus, we are hesitant to place a lot of weight on the RT differences and primarily focus on the hit rate and false alarm results.

Across groups, BNT scores significantly predicted performance on all three outcome measures (although this effect did not differ for LD and HD categorization). This relationship has at least two possible explanations. First, the categorization task, as designed by L\&M, does require some linguistic processing: the participants need to read and understand the label, which often consists of multiple words (e.g., "non-food things found in the kitchen"). Thus, a disruption in receptive language may make the categorization task more difficult for 
individuals with aphasia. Under this explanation, BNT scores may be a proxy for overall aphasia severity. The second explanation is that, due to the proximity of language-specific and multiple-demand brain regions in some parts of the brain (Fedorenko et al., 2012; Fedorenko \& Blank, 2020), brain damage that causes lower BNT scores also leads to difficulties with executively challenging tasks. The categorization task adopted from L\&M involves visual search and selecting among multiple options, which require substantial executive involvement (Petersen \& Posner, 2012; Posner \& Petersen, 1990); thus, categorization difficulties might reflect this increased recruitment of executive demand resources.

Why did we fail to find support for the LD-specific language recruitment hypothesis? One possibility is that the selective impairment in LD categorization manifests only in individuals with low naming performance. Thus, we might have missed the effect of interest because we recruited participants with a fairly wide range of naming scores. To address this potential concern, we conducted a modified version of the categorization experiment with a new set of participants, with a focus on individuals with severe naming impairments in the aphasia group.

\section{Experiment 2}

The aim of Experiment 2 was three-fold. First, we wanted to follow up on the relationship between naming ability (BNT scores) and categorization performance, which was reported by L\&M and found in Experiment 1. Thus, we recruited participants with aphasia who had severe anomia, as measured by the BNT (score range 1-11, compared to 12-57 in Experiment 1; see Tables 1 and 4). Second, we adjusted the paradigm to minimize executive demands, including attention, visual search, selection/inhibition, and updating. Third, we sought to validate a version of the task that could be used in an fMRI setting (time-locked to events). See Figure 1 (bottom) for the modified task setup.

\subsection{Method}

\subsubsection{Participants}

Neurotypical participants $(N=15(15 \mathrm{~F})$, age $M=72.47, S D=6.41)$ were recruited by convenience sampling; patients with chronic aphasia and severe lexical access impairment 
( $N=5$ (all males), age $M=66.60, S D=8.91$ ) were recruited from Aphasia volunteer research registers; PD patients $(N=15$ ( $1 \mathrm{~F})$, age $M=66.60, S D=6.38)$ were recruited from the Parkinson's UK Research Registry (see Table 4 for detailed participant information). None of the participants took part in Experiment 1. All participants used English as their primary language and were offered a $£ 15.00$ reimbursement. Ethical approval was granted by the UCL Research Ethics panel, Project ID: LC/2013/05, and all volunteers gave informed consent to participate in the experiment.

Table 4. Participant information, Experiment 2.

\begin{tabular}{|c|c|c|c|c|c|c|}
\hline Group & Participant & Age & Education & Gender & $\begin{array}{c}\text { TPO } \\
\text { (months) }\end{array}$ & BNT \\
\hline \multirow[t]{15}{*}{ Neurotypical } & 1 & 68 & Degree-Level & $F$ & - & 51 \\
\hline & 2 & 61 & Postgraduate & $F$ & - & 41 \\
\hline & 3 & 85 & Degree-Level & $F$ & - & 54 \\
\hline & 4 & 73 & Postgraduate & $F$ & - & 58 \\
\hline & 5 & 72 & Up to 18 & $F$ & - & 58 \\
\hline & 6 & 77 & Postgraduate & $F$ & - & 55 \\
\hline & 7 & 77 & Degree-Level & $F$ & - & 59 \\
\hline & 8 & 66 & Degree-Level & $F$ & - & 57 \\
\hline & 9 & 66 & Postgraduate & $F$ & - & 54 \\
\hline & 10 & 76 & Degree-Level & $F$ & - & 59 \\
\hline & 11 & 65 & Postgraduate & $F$ & - & 56 \\
\hline & 12 & 80 & Up to 18 & $F$ & - & 45 \\
\hline & 13 & 74 & Postgraduate & $F$ & - & 54 \\
\hline & 14 & 71 & Degree-Level & $F$ & - & 57 \\
\hline & 15 & 76 & Degree-Level & $F$ & - & 47 \\
\hline \multirow[t]{15}{*}{ PD } & 1 & 71 & Degree-Level & $M$ & 24 & 58 \\
\hline & 2 & 78 & Degree-Level & $M$ & 24 & 47 \\
\hline & 3 & 64 & Postgraduate & $M$ & 30 & 48 \\
\hline & 4 & 72 & Postgraduate & $M$ & 18 & 59 \\
\hline & 5 & 54 & Degree-Level & $M$ & 204 & 58 \\
\hline & 6 & 72 & Degree-Level & $M$ & 4 & 48 \\
\hline & 7 & 62 & Postgraduate & $F$ & 120 & 56 \\
\hline & 8 & 65 & Postgraduate & M & 17 & 59 \\
\hline & 9 & 74 & Up to 18 & M & 96 & 56 \\
\hline & 10 & 67 & Up to 16 & $M$ & 60 & 54 \\
\hline & 11 & 67 & Postgraduate & $M$ & 72 & 59 \\
\hline & 12 & 59 & Postgraduate & $M$ & 30 & 58 \\
\hline & 13 & 59 & Degree-Level & $M$ & 48 & 60 \\
\hline & 14 & 67 & Degree-Level & $M$ & 18 & 55 \\
\hline & 15 & 68 & Degree-Level & $M$ & 98 & 48 \\
\hline \multirow[t]{5}{*}{ Aphasia } & 1 & 58 & Up to 18 & $M$ & 42 & 5 \\
\hline & 2 & 68 & Up to 16 & $M$ & 68 & 9 \\
\hline & 3 & 77 & Up to 18 & M & 111 & 11 \\
\hline & 4 & 57 & Degree-Level & $M$ & 34 & 1 \\
\hline & 5 & 73 & Up to 18 & $M$ & 326 & 4 \\
\hline
\end{tabular}

TPO- Time Post Onset; BNT- Boston Naming Test

\subsubsection{Design and Materials}


The categories were identical to those of Experiment 1. The images were also largely the same although some were replaced by better quality photographs. Unlike Experiment 1, we presented the images sequentially (Figure 2, bottom). Each block started with a category label, followed by 12 images presented one at a time. The category label remained on the screen to minimize memory demands. The images for each category block were randomly selected from the general set of pictures for that category. The number of targets varied across blocks (minimum: 4, maximum: 6) so as to minimize the implicit learning of a fixed number of targets, which could have incentivized participants to keep track of the total number of targets and thereby increase their cognitive load. Categories were grouped by dimension (LD/HD) into groups of 4 , for a total of 8 blocks (4 blocks per dimension). These 8-block sequences ("runs") were separated by a rest period of fixation (10s in duration). The order of runs, the order of conditions within runs (LD first vs. HD first), the order of categories within runs, and the order of images within category blocks were randomized for each participant.

\subsubsection{Experimental Procedure}

Testing was carried out individually either in a quiet well-lit room at a clinic nearest to the participant's location or in their home, using a Dell Latitude E5540 (14.1-inch display). The experiment was set up using Python (version 2.7.10). Each category block started with an instruction screen presented for $2 \mathrm{~s}$ that read 'Please find [CATEGORY LABEL]' (e.g., 'Please find objects that hold water'). Given that the participants in the aphasia group were severely lexically impaired and had difficulty processing orthographic information, the experimenter read the category label aloud to all participants (in all groups) during this trialinitial $2 \mathrm{~s}$ window. This screen was followed by a sequence of 12 images presented one at a time for a maximum of 10s per image. For each image, participants had to decide whether the depicted object belong to the target category by pressing one of two keys on the keyboard: the ' $\mathrm{Y}$ ' key marked with a green sticker for YES, or the ' $N$ ' key marked with a red sticker for NO. If no response was recorded for $10 \mathrm{~s}$, the experiment advanced to the next image. Responses and response times were recorded for each image. The experiment lasted approximately 1 hour. The BNT was administered at the beginning of the testing session.

\subsubsection{Statistical analyses}

The statistical analysis procedure was the same as in Experiment 1. No trials were excluded. 


\subsection{Results}

\subsubsection{Group profiles}

As expected, the groups differed significantly in their BNT scores $(F(2,32)=202.67, p<.001)$. Post-hoc pairwise comparisons revealed that the BNT scores of participants with aphasia $(M=6.00, S D=4.00)$ were significantly lower than both neurotypical participants $(p<.001)$ and participants with PD $(p<.001)$, with the latter two groups not differing significantly $(M=53.67, S D=5.42$ vs. $M=54.87, S D=4.73, p>.999)$. The groups did not differ in age $(F(2,32)=3.23, p=.053)$, but a significant difference was observed in the level of education $(F(2,32)=5.42, p=.009)$, with neurotypical participants and participants with PD having significantly more years of education than participants with aphasia $(p=.010$ and $p=.016$, respectively). The neurotypical participants and participants with PD did not differ $(p>.999)$.

\subsubsection{Categorization task}

As in Experiment 1, three dependent variables were analyzed: hit rate, false alarm rate, and RT. Categorization results are summarized in Figure 3.

Hit rate. Similar to the results of Experiment 1, participants with aphasia had similar hit rates for LD categories $(M=0.88, S D=0.08)$ and HD categories $(M=0.91, S D=0.06$; LD>HD: $\beta=-$ 0.34, $S E=0.29, p=.252)$. Participants with aphasia had overall lower hit rates $(M=0.90$, $S D=0.07)$ compared to neurotypical participants $(M=0.97, S D=0.02$; neurotypical $>$ aphasia: $\beta=1.45, S E=0.32, p<.001)$ and participants with $\mathrm{PD}(M=0.95, S D=0.03$; PD>aphasia: $\beta=0.86$, $S E=0.31, p=.005$ ), which is consistent with Experiment 1's negative relationship between naming ability and categorization performance. We did not observe a reliable category dimension by group interaction for the aphasia vs. neurotypical comparison $(\beta=0.44$, $S E=0.26, p=.086)$, nor for the aphasia vs. PD comparison $(\beta=0.42, S E=0.23, p=.070)$. Follow-up analysis showed that there was no main effect of category dimension across groups $(\beta=0.05, S E=0.25, p=.990)$, nor within the neurotypical group $(\beta=-0.11, S E=0.30$, $p=.960)$, or the PD group ( $\beta=-0.08, S E=0.28, p=.975$ ). Overall, the group comparison of hit rate (Figure 3A) does not support the LD-specific language recruitment hypothesis. 
A

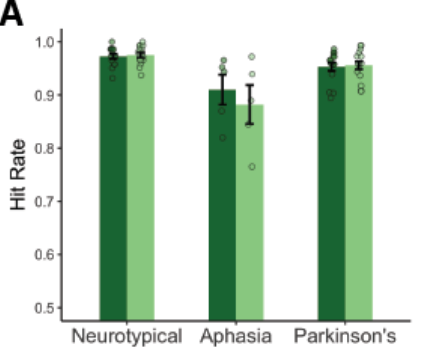

D

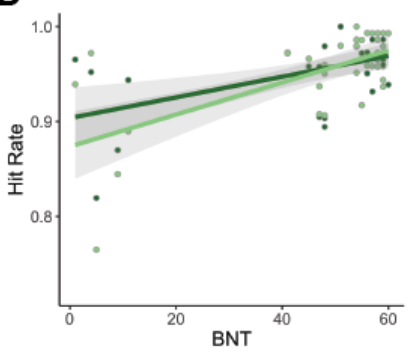

B

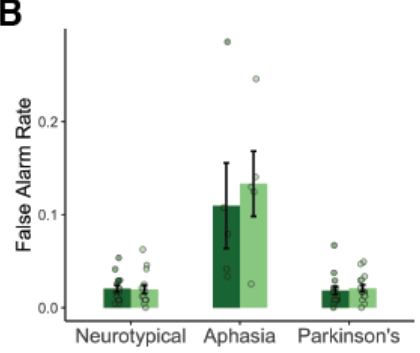

E

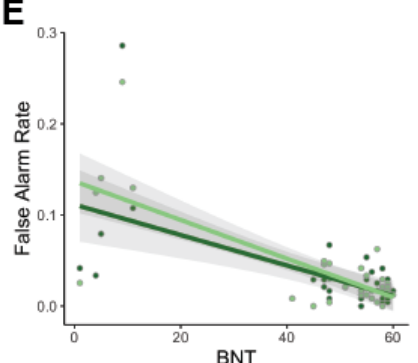

C

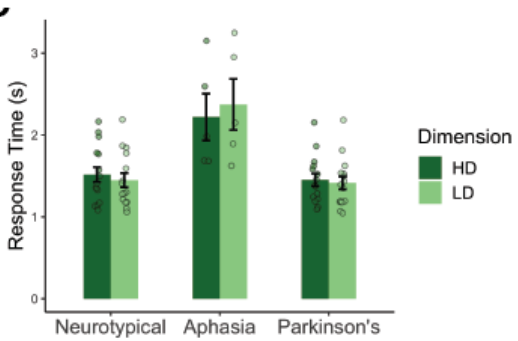

$\mathbf{F}$

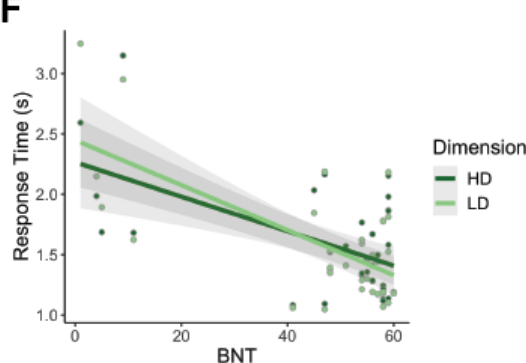

Figure 3. Experiment 2 results. (A) Hit Rate, (B) False Alarm Rate, and (C) Response Time $(R T)$ across the three participant groups (here, $R T$ is the time until participants pressed a “yes" or "no" button for each image within a trial). (D) Hit Rate, (E) False Alarm Rate, and (F) RT plotted against participants' BNT scores, a measure of naming performance.

False alarm rate. The false alarm rate results (Figure 3B) were consistent with the hit rate results. Participants with aphasia had comparable false alarm rates for LD categories $(M=0.13, S D=0.08)$ and HD categories $(M=0.11, S D=0.10 ; \mathrm{LD}>\mathrm{HD}: \beta=0.13, S E=0.27$, $p=.626)$. The overall false alarm rate among participants with aphasia $(M=0.12, S D=0.09)$ was higher than in neurotypical participants $(M=0.02, S D=0.02$; neurotypical $>$ aphasia: $\beta=-$ 1.91, $S E=0.32, p<.001)$ and participants with PD $(M=0.02, S D=0.02$; PD>aphasia: $\beta=-1.94$, $S E=0.32, p<.001)$. The group by category dimension interactions were not significant for either the neurotypical vs. aphasia comparison $(\beta=-.38, S E=.21, p=.075)$, nor the PD vs. aphasia comparison $(\beta=-0.19, S E=0.22, p=.381)$. Follow-up analyses showed no effect of category dimension across groups $(\beta=0.06, S E=0.25, p=.985)$, nor within the neurotypical group $(\beta=0.25, S E=0.29, p=.732)$ or the PD group $(\beta=0.06, S E=0.29, p=.990)$.

Response time. RTs in Experiment 2 were the only measure where the pattern was consistent with the LD-specific language recruitment hypothesis. Participants with aphasia were slower to respond during LD trials $(M=2.37, S D=0.70)$ compared to HD trials $(M=2.22, S D=0.64$; LD>HD: $\beta=0.16, S E=0.08, p=.044)$. The overall RTs for participants with aphasia $(M=2.30$, $S D=0.64)$ were longer than for neurotypical participants $(M=1.48, S D=0.34 ; \beta=-.81$, 
$\mathrm{SE}=0.19, p<.001)$ and participants with $\mathrm{PD}(M=1.43, S D=0.29 ; \beta=-0.86, S E=0.19, p<.001)$.

We also observed an interaction between group and category dimension for both the neurotypical vs. aphasia comparison $(\beta=-0.23, S E=0.03, p<.001)$ and the PD vs. aphasia comparison $(\beta=-0.19, S E=0.03, p<.001)$, such that participants with aphasia had longer RTs for LD categories compared to HD categories. Follow-up analyses showed no overall effect of category dimension across groups $(\beta=-0.02, S E=0.07, p=.985)$, within the neurotypical group $(\beta=0.07, S E=0.07, p=.669)$ or within the PD group $(\beta=.04, S E=0.07, p=.907)$, suggesting that the LD vs. HD difference was specific to participants with aphasia. Note, however, that the effect size of the interactions is much smaller than the overall differences between the aphasia group and the two control groups (Figure 3C).

Effect of naming performance. To explore the effect of naming ability on categorization performance in this revised version of the task, we again fitted mixed effect regression models with BNT scores, category dimension, interaction between BNT and category dimension, and education level as fixed effects, and participants (across the three groups) and categories as random effects. We found that BNT still significantly predicted all dependent variables (hit rate: $\beta=0.38, S E=0.13, p=.002$; false alarm rate: $\beta=-0.58, S E=0.12, p<.001$; RT: $\beta=-0.29, S E=0.07, p<.001)$. As in Experiment 1, this model did not reveal a main effect of category dimension (hit rate: $\beta=0.04, S E=0.26, p=.884$; false alarm rate: $\beta=-0.12, S E=0.26$, $p=.639$; RT: $\beta=-0.02, S E=0.07, p=.742$ ); however, unlike Experiment 1, we observed an interaction between $\mathrm{BNT}$ and category dimension for hit rate $(\beta=0.16, S E=0.08, p=.039)$ and RT $(\beta=-0.08, S E=0.01, p<.001)$. We did not observe such an interaction for false alarm rate ( $\beta=-0.11, S E=0.07, p=.090)$. Finally, education was no longer a significant predictor for any dependent variable (hit rate: $\beta=0.11, S E=0.15, p=.484$; false alarm rate: $\beta=-0.20, S E=0.15$, $p=.190 ; \mathrm{RT}: \beta=-0.01, S E=0.08, p=.940)$.

Single case analysis. Scrutiny of individual participants' scores casts some doubt on the relationship between BNT and categorization performance. Specifically, participant A4 in the aphasia group (Table 4) had a very low BNT score (1/60), but nonetheless performed very well relative to both the neurotypical and PD groups (hit rate: LD 94\%, HD 97\%; false alarm rate: $\mathrm{LD} 2.54 \%, \mathrm{HD} 4.17 \%$; all results are within $2 \mathrm{SD}$ of mean performance in the neurotypical group). This dissociation indicates an absence of a direct causal relationship between naming and categorization. 


\subsection{Interim discussion}

Experiment 2 had several goals, including an additional attempt to replicate differences between $\mathrm{LD}$ and $\mathrm{HD}$ categorization, investigating the effect of severe naming impairments on categorization performance, testing whether the LD-specific language recruitment hypothesis may find support in a paradigm modified to reduce executive demands, and validating a paradigm for use in fMRI. We discuss our results below.

We did not find evidence that LD categorization is overall more challenging than $\mathrm{HD}$ categorization: no significant differences were observed between LD and HD categories for any of the performance measures (hit rate, false alarm rate, and RT). This result is consistent with Experiment 1 and fails to replicate the results of L\&M, who report lower performance on LD categorization across groups. However, this finding does not necessarily undermine the critical claim of the LD-specific language recruitment hypothesis, namely, that participants with aphasia would exhibit a selective impairment in LD categorization.

As in Experiment 1, naming ability significantly predicted performance on all three outcome measures. Furthermore, because in this experiment we specifically recruited participants with aphasia who had poor naming performance, we also observed a group difference: participants with aphasia had lower hit rates, more false alarms, and longer response times than the two control groups. This evidence points to a link between naming performance and categorization. However, as in Experiment 1, this link might arise from the fact that task instructions are presented verbally; thus, linguistic impairments might affect task performance simply because they make it more challenging to process the instructions.

Another reason to be skeptical of a direct link between naming and categorization is an instance of dissociation between these two tasks: participant A4 had a BNT score of 1, and yet performed similar to controls on the categorization task. Dissociation cases are critical in informing debates about cognitive architecture in general and about the role of language in enabling other cognitive abilities in particular (e.g., Caramazza \& Coltheart, 2006). Naturally occurring brain lesions do not respect the boundaries between functionally distinct brain areas, and comorbidities or associations of impairments are common (e.g., E. Bates et al., 2003). For example, damage to the left inferior frontal gyrus is likely to cause multiple cognitive impairments due to a high functional heterogeneity of that region (Fedorenko et al., 2012; Fedorenko \& Blank, 2020). Thus, a correlation we observe between naming ability and 
categorization might be caused by the fact that the regions supporting these functions are located nearby (rather than by the fact that these two functions are supported by the same brain region/mechanism). The dissociation we observe in participant A4 supports this possibility and suggests that the language-categorization link might be caused by anatomical coincidence rather than by cognitive interdependency. In any case, it is clear that in some cases, severely limited lexical access does not prevent success on the categorization task, revealing that intact linguistic skills are not necessary for categorizing objects.

One of the primary goals of Experiment 2 was to establish whether the putative effect of language on LD categorization might manifest itself more clearly and consistently if the categorization task is modified to reduce the overall cognitive load. We did not find this effect when looking at response accuracies: participants with aphasia did not show a selective impairment in hit rate nor a selective increase in false alarm rate for LD categories. However, we did observe an interaction between RT and category dimension, such that participants with aphasia had longer RTs for LD categories compared to HD categories. We also observed an interaction between naming ability and category dimension, such that lower BNT scores were associated with lower hit rate on LD categories more so than on HD categories, and with longer RTs on LD categories more so than on HD categories. This pattern is consistent with the LD-specific language recruitment hypothesis; however, the fact that we did not observe such a pattern in Experiment 1, which followed L\&M's design and procedure more closely, suggests that this effect is not stable and varies depending on the makeup of the aphasia group and, possibly, the details of the experimental setup. Further, the group by category dimension interaction was absent for the false alarm rate. Given that, in Experiment 1, the interaction effect for the false alarm rate went in the opposite direction from that predicted by the LD-specific recruitment hypothesis, we find it difficult to reconcile the results from the two experiments with that hypothesis.

The results of Experiments 1 and 2 did not allow us to definitively answer the question of whether language plays a key role in LD categorization. Group comparisons in both experiments failed to replicate the selective LD categorization impairments as reported in L\&M; moreover, in Experiment 1, the effect of aphasia on false alarm rate was actually higher for HD categories. On the other hand, Experiment 2 did show a selective decrease in hit rate (and increase in RTs) for LD categories in participants with low naming scores, as 
predicted by the LD-specific language recruitment hypothesis. However, even this piece of evidence is undermined by the dissociation case of participant A4.

To definitively establish whether LD categorization recruits the language system, we next turned to fMRI.

\section{Experiment 3}

To test the relationship between language and categorization at a neural level, we conducted an fMRI experiment. In this experiment, neurotypical participants performed the same LD/HD categorization task as participants in Experiment 2, as well as two localizer tasks used to identify the networks of interest: the language network, which responds selectively to linguistic input (Braga et al., 2020; Fedorenko et al., 2010, 2011), and the multiple demand network, which is sensitive to general cognitive effort and implicated in executive functions (Assem, Glasser, et al., 2020; Duncan, 2010; Fedorenko et al., 2013). Examining activation patterns in both the language and the multiple demand networks allows us to examine their contributions to $\mathrm{LD}$ and HD categorization.

As discussed before, brain damage leading to aphasia is often comorbid with multiple demand network damage: the language-selective regions and these domain-general regions in left inferior frontal cortex lie in close proximity to each other (Blank et al., 2014; Fedorenko et al., 2012; Fedorenko \& Blank, 2020), with precise locations varying substantially across individuals. Thus, impaired categorization performance of participants with aphasia in Experiments 1 and 2 could have potentially arisen from damage to either network (or to both). Experiment 3 allows us to disambiguate between these possibilities. If, as suggested by L\&M, LD categorization indeed relies on language more than HD categorization, we expect to see more activity within the language system during LD trials compared to HD trials. Further, if LD categorization is a more cognitively demanding task, we expect to see higher responses within the multiple demand network during LD trials compared to HD trials (in accordance with the fact that multiple demand regions are sensitive to effort across diverse tasks; Duncan \& Owen, 2000; Fedorenko et al., 2013; Hugdahl et al., 2015). Finally, if a brain network does not respond to either LD or HD categorization, we can conclude that this network is not recruited for this task. 


\subsection{Method}

\subsubsection{Participants}

Fourteen neurotypical participants ( $7 \mathrm{~F}$, age $M=22.31, S D=3.51)$ were recruited from MIT and the surrounding community and paid $\$ 60$ for their participation. All were native speakers of English. One participant was left-handed (see Willems et al., 2014, for motivation to include left-handers in cognitive neuroscience research) but showed typical left-lateralized language activations as determined by the language localizer task (described below). All participants gave informed consent in accordance with the requirements of MIT's Committee On the Use of Humans as Experimental Subjects (COUHES).

\subsubsection{Design, materials, and procedure}

Each participant completed a language localizer task aimed at identifying languageresponsive brain regions (Fedorenko et al., 2010), a spatial working memory (WM) task aimed at identifying the multiple demand network (Fedorenko et al., 2013), and the critical categorization task. Some participants completed one or more additional tasks for unrelated studies. The entire scanning session lasted two hours.

Language network localizer. Participants read sentences (e.g., NOBODY COULD HAVE PREDICTED THE EARTHQUAKE IN THIS PART OF THE COUNTRY) and lists of unconnected, pronounceable nonwords (e.g., U BIZBY ACWORRILY MIDARAL MAPE LAS POME U TRINT WEPS WIBRON PUZ) in a blocked design. Each stimulus consisted of twelve words/nonwords. The sentences > nonword-lists contrast has been previously shown to reliably activate high-level language processing regions and to be robust to changes in the materials, task, and modality of presentation (Chen et al., in prep.; Fedorenko et al., 2010; Mahowald \& Fedorenko, 2016; Scott et al., 2017). For details of how the language materials were constructed, see Fedorenko et al. (2010). The materials are available at http://evlab.mit.edu/funcloc. Stimuli were presented in the center of the screen, one word/nonword at a time, at the rate of $450 \mathrm{~ms}$ per word/nonword. Each stimulus was preceded by a $100 \mathrm{~ms}$ blank screen and followed by a $400 \mathrm{~ms}$ screen showing a picture of a finger pressing a button, and a blank screen for another $100 \mathrm{~ms}$, for a total trial duration of $6 \mathrm{~s}$. Participants were asked to press a button whenever they saw the picture of a finger pressing a button. This task was included to help participants stay alert and awake. Condition order was counterbalanced across runs. Experimental blocks lasted 18s (with 3 trials per block), and 
fixation blocks lasted 14s. Each run (consisting of 5 fixation blocks and 16 experimental blocks) lasted 358s. Each participant completed 2 runs.

Multiple demand network localizer. Participants had to keep track of four (easy condition) or eight (hard condition) sequentially presented locations in a 3×4 grid (Fedorenko et al., 2013). The hard > easy contrast has been previously shown to robustly activate multiple demand regions (Assem, Blank, et al., 2020; Blank et al., 2014; Fedorenko et al., 2013; Mineroff et al., 2018). Stimuli in both conditions were presented in the center of the screen across four steps. Each of these steps lasted for 1s and presented one location on the grid in the easy condition, and two locations in the hard condition. Each stimulus was followed by a choiceselection step, which showed two grids side by side. One grid contained the locations shown on the previous four steps, while the other contained an incorrect set of locations. Participants were asked to press one of two buttons to choose the grid that showed the correct locations. Condition order was counterbalanced across runs and participants. Experimental blocks lasted 32s (with 4 trials per block), and fixation blocks lasted 16s. Each run lasted 448s, consisting of 12 experimental blocks (6 per condition), and 4 fixation blocks. Twelve participants completed two runs and two participants completed one run.

Critical categorization task. The categorization materials were the same as those used in Experiment 2 (see Figure 1, bottom). The timing differed in the following way. In order to make blocks uniform in duration, each category block started with a category label presented for $2 \mathrm{~s}$, and then the 12 images were presented sequentially at the fixed speed of $2 \mathrm{~s}$ per image. As in Experiment 2, any given category block contained between 4 and 6 target images. Participants were asked to press a button if the picture belonged to the target category and not to press anything if it did not. As before, the category label was displayed at the top of the screen for the duration of the trial to minimize memory demands. Category blocks lasted $26 \mathrm{~s}$ ( $2 \mathrm{~s}$ category label presentation $+2 \mathrm{~s} * 12$ images), and fixation blocks lasted 14s. Each run, consisting of 12 category blocks (6 LD and 6 HD) and 4 fixation blocks, lasted 368s. Each participant completed 3 runs. Across the 3 runs, any given participant saw a random subset of the 32 categories, with some categories repeating (but never repeating within a run; see Appendix 1 Table 1 for details). Condition order was counterbalanced across runs and participants. 


\subsection{3 fMRI data acquisition}

Structural and functional data were collected on the whole-body, 3 Tesla, Siemens Trio scanner with a 32-channel head coil, at the Athinoula A. Martinos Imaging Center at the McGovern Institute for Brain Research at MIT. T1-weighted structural images were collected in 176 sagittal slices with $1 \mathrm{~mm}$ isotropic voxels $(\mathrm{TR}=2530 \mathrm{~ms}$, TE=3.48ms). Functional, blood oxygenation level dependent (BOLD), data were acquired using an EPI sequence (with a $90^{\circ}$ flip angle and using GRAPPA with an acceleration factor of 2), with the following acquisition parameters: thirty-one $4 \mathrm{~mm}$ thick near-axial slices acquired in the interleaved order (with $10 \%$ distance factor), $2.1 \mathrm{~mm} \times 2.1 \mathrm{~mm}$ in-plane resolution, FoV in the phase encoding $(\mathrm{A}>>\mathrm{P})$ direction $200 \mathrm{~mm}$ and matrix size $96 \mathrm{~mm} \times 96 \mathrm{~mm}$, TR=2000ms and $\mathrm{TE}=30 \mathrm{~ms}$. The first $10 \mathrm{~s}$ of each run were excluded to allow for steady state magnetization.

\subsection{4 fMRI data preprocessing}

MRI data were analyzed using SPM12 and custom MATLAB scripts (available in the form of an SPM toolbox from https://evlab.mit.edu/funcloc/). Each participant's data were motion corrected and then normalized into a common brain space (the Montreal Neurological Institute (MNI) template) and resampled into $2 \mathrm{~mm}$ isotropic voxels. The data were then smoothed with a 4mm FWHM Gaussian filter and high-pass filtered (at 200s). Effects were estimated using a General Linear Model (GLM) in which each experimental condition was modeled with a boxcar function (modeling entire blocks) convolved with the canonical hemodynamic response function (HRF).

\subsubsection{Defining individual functional regions of interest (fROIs)}

Responses to the critical categorization experiment were extracted from regions of interest that were defined functionally in each individual participant (Nieto-Castañón \& Fedorenko, 2012; Saxe et al., 2006). Three sets of functional regions of interest (fROIs) were definedone for the language network, one for the multiple demand network, and one for the putative LD $>$ HD categorization regions. To do so, we used the Group-constrained Subject-Specific (GSS) approach (Fedorenko et al., 2010; Julian et al., 2012). In particular, fROIs were constrained to fall within a set of "parcels", which marked the expected gross locations of activations for the relevant contrast. For the language network, the parcels were generated based on a group-level representation of language localizer data from 220 participants. For the multiple demand network, the parcels were generated based on a group-level representation of spatial working memory task data from 197 participants. For the putative 
LD categorization regions, we generated the parcels based on the data collected in this study. The parcels are available on OSF (https://osf.io/guwh8/). To create each set of parcels, individual activation maps for the relevant localizer contrast were binarized (by turning all voxels significant at the $p<.001$ whole-brain threshold (uncorrected) into 1s, and the rest into 0s) and overlaid in the MNI space to create a probabilistic overlap map. Note that for the multiple demand network, the individual activation maps were averaged across the two hemispheres prior to binarizing. The map was then smoothed (FWHM $=6 \mathrm{~mm})$, and voxels with fewer than $10 \%$ of participants overlapping were excluded. The resulting map was divided into regions using a watershed algorithm. Finally, we excluded parcels that did not show significant effects for the relevant localizer contrast in a left-out run or did not contain supra-threshold voxels in at least $60 \%$ of the participants (for language and multiple demand networks) or in at least $50 \%$ of the participants (for putative LD categorization regions). For the multiple demand network, we also a) excluded parcels in the visual cortex (the hard condition includes more visual information than the easy condition and thus yields more activation in the visual cortex), and b) divided a parcel that encompassed parts of both the precentral gyrus and the opercular portion of the inferior frontal gyrus according to the macroanatomical boundary.

For each participant, each set of masks was intersected with the participant's activation map for the relevant contrast (sentences>nonwords for the language network, hard>easy spatial $\mathrm{WM}$ for the multiple demand network, and LD>HD for putative LD categorization regions). Within each mask, the voxels were sorted based on their $t$ values for the relevant contrast, and the top $10 \%$ of voxels were selected as that participant's fROI. This top n\% approach ensures that the fROIs can be defined in every participant, thus enabling us to generalize the results to the entire population (Nieto-Castañón \& Fedorenko, 2012).

\subsubsection{Examining the functional response profiles of fROIs}

After defining fROIs in individual participants, we evaluated their responses to the conditions of interest by averaging the responses across voxels to get a single value per condition per fROI. The responses to the localizer conditions (sentences and nonwords for language fROIs, hard and easy working memory conditions for multiple demand fROIs, and LD and HD categorization for categorization fROIs) were estimated using an across-runs cross-validation procedure, where one run was used to define the fROI and the other to estimate the response magnitudes, then the procedure was repeated switching which run was used for fROI 
definition vs. response estimation, and finally the estimates were averaged to derive a single value per condition per fROI per participant. This cross-validation procedure allows one to use all of the data for defining the fROIs as well as for estimating their responses (see NietoCastañón \& Fedorenko, 2012, for discussion), while ensuring the independence of the data used for fROI definition and response estimation (Kriegeskorte et al., 2009). Two participants completed only one run of the multiple demand localizer task; therefore, we did not estimate the strength of their responses to the hard and easy multiple demand localizer conditions but ensured that the whole-brain activation maps for the hard>easy contrast looked as expected.

\subsubsection{Statistical analyses}

Similar to Experiments 1 and 2, we analyzed our data using mixed effect regression models (Baayen et al., 2008). For hit rate and false alarms we use logistic regression (Jaeger, 2008). For RT and fROIs response magnitudes, we use linear regression. In all models, condition was a fixed effect and participant was a random intercept. The model for the multiple demand network included hemisphere as an additional fixed effect. For language and multiple demand network analyses, we also included fROI as a random intercept and then ran followup analyses on individual fROIs using false discovery rate (FDR) correction (Benjamini \& Hochberg, 1995) for the number of fROIs in each network. Behavioral analyses used sum coding for condition (LD vs. HD in the categorization task and Hard vs. Easy in the multiple demand localizer task). Neuroimaging analyses used custom contrasts (see Appendix 2 for detailed contrast specification). The mixed effect analyses were run using the lmer function from the lme4 R package (D. Bates et al., 2015); statistical significance of the effects was evaluated using the lmerTest package (Kuznetsova et al., 2017). The hypotheses-specific contrasts were defined using the hypr package (Rabe et al., 2020).

If linguistic resources are engaged during categorization, we would expect an overall high response of the language network to categorization conditions. Further, if, as L\&M have argued, LD categorization taxes linguistic resources to a greater extent, we would expect to see stronger response of this network to the LD compared to the HD condition. Lastly, if LD categorization is generally more taxing, we would expect to see greater responses to the LD condition in the domain-general multiple demand regions that are sensitive to effort across diverse tasks (Duncan, 2010, 2013; Fedorenko et al., 2013; Hugdahl et al., 2015). 


\subsection{Results}

\subsubsection{Behavioral data}

Multiple demand network localizer. Due to a technical error, behavioral data for one participant got overwritten. For the remaining thirteen participants, performance on the spatial working memory task was as expected: participants were more accurate and faster in the easy condition (accuracy $M=93.91 \%, S D=3.00 \%$; reaction time (RT)=1.18s, $S D=0.16 \mathrm{~s}$ ) than the hard condition (accuracy $M=79.65 \%, S D=12.03 \%$; RT $M=1.52 \mathrm{~s}, S D=0.25 \mathrm{~s}$ ). Mixed effect models with condition as a fixed effect and participant as a random intercept showed that both accuracy and RT effects were significant (accuracy: $\beta=-1.41, \mathrm{SE}=0.202, p<.001$; $\mathrm{RT}: \beta=0.33, S E=0.027, p<.001)$.

Critical categorization task. The hit rate for the two categorization conditions was not significantly different (LD $M=93.62 \%, S D=9.84 \%$; HD $M=93.42 \%, S D=8.65 \%$; LD $>\mathrm{HD}$ $\beta=0.03, S E=0.22, p=.897)$. Similarly, the number of false alarms did not significantly differ across conditions (LD $M=3.01 \%, S D=1.43 \%$; $\mathrm{HD} M=3.34 \%, S D=2.39 \%$; $\mathrm{LD}>\mathrm{HD} \beta=-0.37$, $S E=0.38, p=.329)$. Finally, there was no significant difference between response times in the $\mathrm{LD}$ condition $(\mathrm{RT}=0.81 \mathrm{~s}, S D=0.1 \mathrm{~s})$ and the $\mathrm{HD}$ condition $(\mathrm{RT}=0.84 \mathrm{~s}, S D=0.1 \mathrm{~s} ; \mathrm{LD}>\mathrm{HD} \beta=-$ $0.03, S E=0.02, p=.156)$.
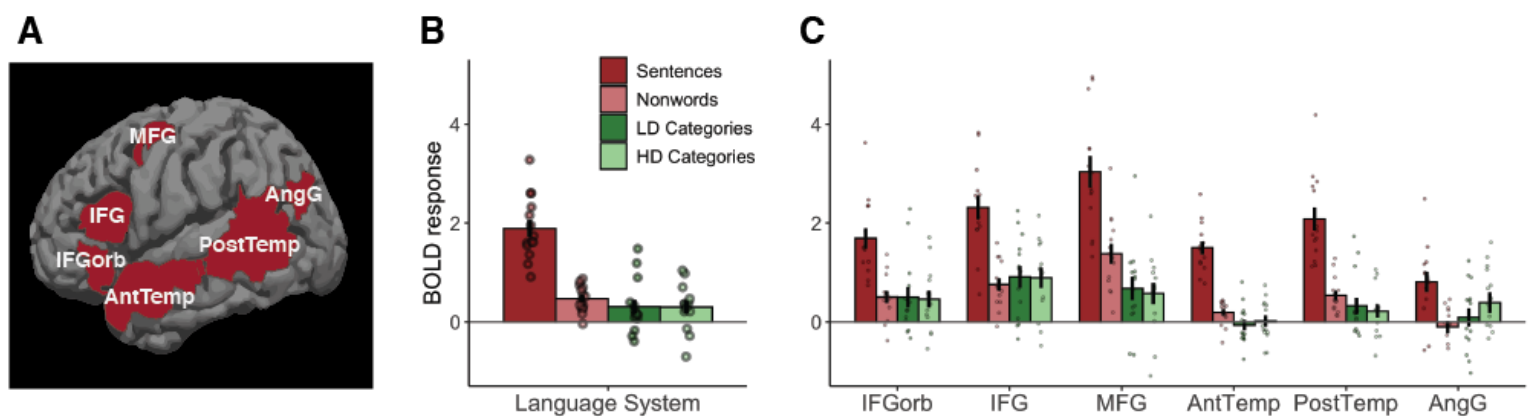

Figure 4. Categorization responses within the language brain network. (A) Parcels used to define functional regions of interest (fROIs) in individual participants. (B) Average responses within the language network to four conditions of interest (sentence reading and nonword reading $v s . L D$ and $H D$ categorization). (C) fROI responses to the four conditions of interest.

\subsubsection{Functional response profile of the language network}


Although the sentence reading condition elicited strong responses in the language fROI, the responses to the categorization task were only marginally above $0(\beta=0.42, S E=0.19, p=.054$; see Figure 4), not significantly different from responses to nonword reading $(\beta=0.13$, $S E=0.09, p=.144)$, and significantly weaker than responses to sentences $(\beta=-1.49, S E=.09$, $p<.001)$. Further, there was no significant difference between responses to LD and HD categorization $(\beta=-0.02, S E=0.10, p=.848)$.

Follow-up analyses in individual language fROIs (Appendix 2 Table 1) showed that responses to categorization were significantly above 0 in frontal fROIs (MFG, IFG, and IFGorb). However, none of the responses were significantly higher than responses during the control task, nonword reading, indicating that these responses are not language-specific. Thus, our results suggest that the language network is not involved in either LD or HD categorization in neurotypical participants.

\subsubsection{Functional response profile of the multiple demand network}

Multiple demand network responses to categorization were significantly above $0(\beta=1.07$, $S E=0.21, p<.001$; see Figure 5) and stronger than responses to control conditions from the language localizer task (categorization > sentences: $\beta=0.73, S E=0.08, p<.001$; categorization $>$ nonwords: $\beta=0.41, S E=0.08, p<.001)$. However, they were weaker than responses to the spatial working memory task $(\beta=-1.43, S E=.07, p<.001)$, indicating that the working memory task was more effortful. The responses to the categorization task were stronger in the left hemisphere $(\beta=0.24, S E=0.09, p=.005)$. We also observed an interaction between the working memory > categorization contrast and hemisphere $(\beta=0.29, S E=0.13, p=.024)$, showing that the working memory task engages the right hemisphere to a greater extent. There was also an interaction between the Hard>Easy working memory task and hemisphere, such that the effect was greater in right hemisphere $(\beta=0.38, S E=0.19, p=.040)$.

Importantly, there was a small but significant difference between responses to LD and HD categorization tasks $(\beta=0.19, S E=0.09, p=.025)$, indicating that LD categorization is slightly more effortful than HD categorization in neurotypical participants. 

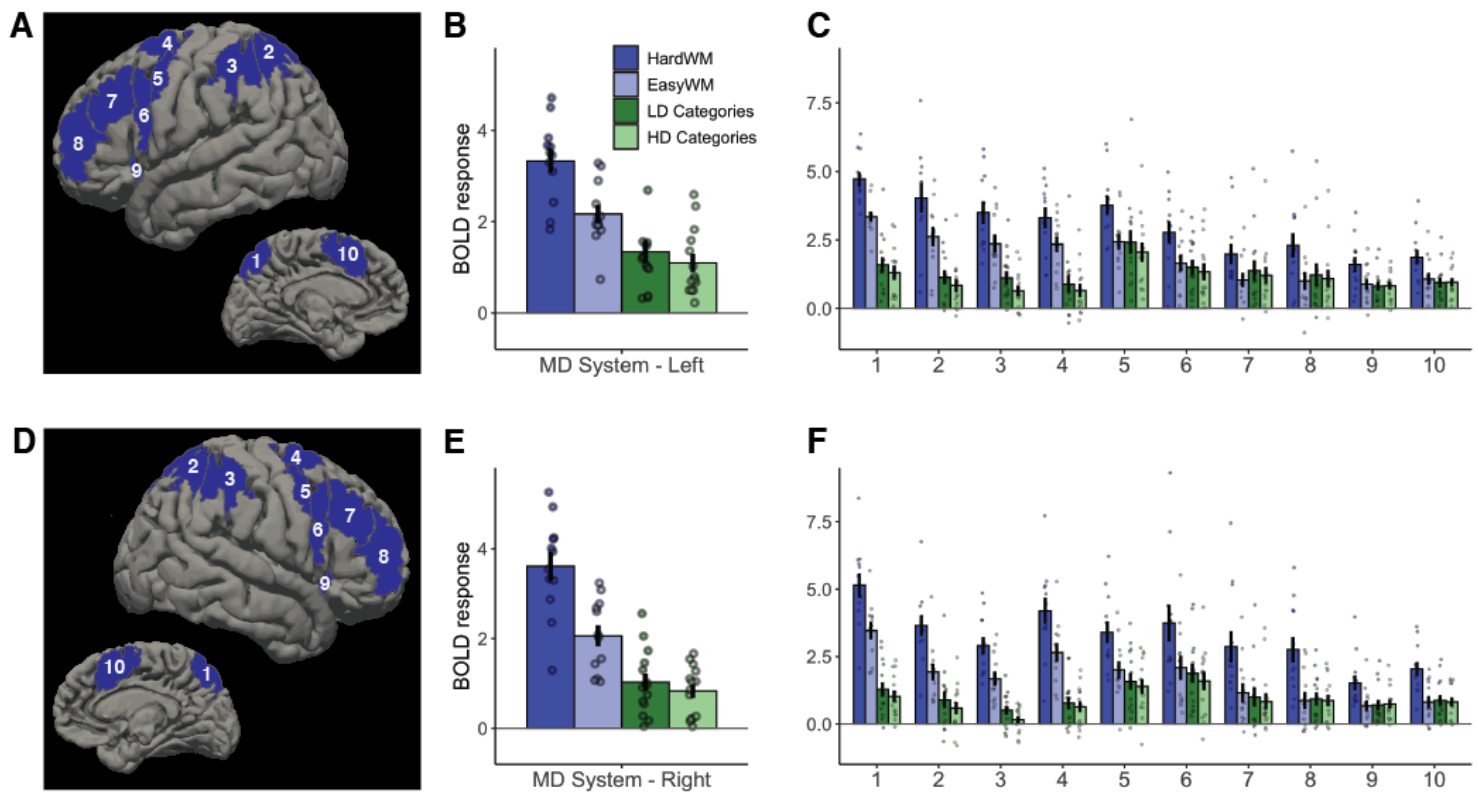

Figure 5. Categorization responses within the multiple demand brain network. (A) Left hemisphere parcels used to define functional regions of interest (fROIs) in individual participants. (B) Average responses within the left hemisphere fROIs to four conditions of interest (hard and easy working memory tasks vs. LD and HD categorization). (C) Left hemisphere fROI responses to the four conditions of interest. (D-F) Parcels, average responses, and fROI-level responses in the right hemisphere.

Follow-up analyses on individual fROIs (Appendix 2 Table 2) showed that responses to categorization were significantly above 0 in all fROIs. However, they were weaker than the overall responses to the working memory task in almost all fROIs (except left middle frontal fROI). This result highlights the domain-general nature of these responses. Further, none of the fROIs had significantly different responses to LD and HD categories, despite the presence of this effect in the network-level analysis.

\subsubsection{Whole-brain analyses}

We also conducted a whole-brain analysis to identify fROIs that might respond more strongly to $\mathrm{LD}$ or HD categorization but lie outside the language and multiple demand fROIs described above. The GSS analysis (see Methods for details) revealed that no regions exhibited consistent HD>LD responses across participants; however, the LD>HD contrast revealed two parcels, both located in left parietal lobe (Figure 6). Further analysis of fROIs defined within these parcels showed that the LD>HD response only reached significance in fROI $2(\beta=0.43, S E=0.17, p=.013)$, but not in fROI $1(\beta=0.58, S E=0.30, p=.060)$. The overall 
categorization response was significantly above 0 in fROI $1(\beta=0.65, S E=0.19, p=.001)$ but not fROI $2(\beta=-0.13, S E=0.15, p=.389)$.

Importantly, both fROIs responded to the working memory task more strongly than to the categorization task (fROI 1: $\beta=1.66, S E=0.21, p<.001$; fROI 2: $\beta=0.64, S E=0.12, p<.001$ ), indicating that these regions likely respond to general cognitive effort rather than to LD categorization (or feature selection) specifically. Neither of the two fROIs exhibited a sentences $>$ nonwords effect (fROI 1: $\beta=-0.51, S E=0.30, p=.094$; fROI 2: $\beta=-0.28, S E=0.17$, $p=.098$ ), which shows that these regions do not respond to linguistic input.

The whole-brain analysis provides additional evidence against the LD-specific language recruitment hypothesis and shows that differences in $\mathrm{LD}$ vs. HD categorization, if present, are likely caused by domain-general mechanisms.

A

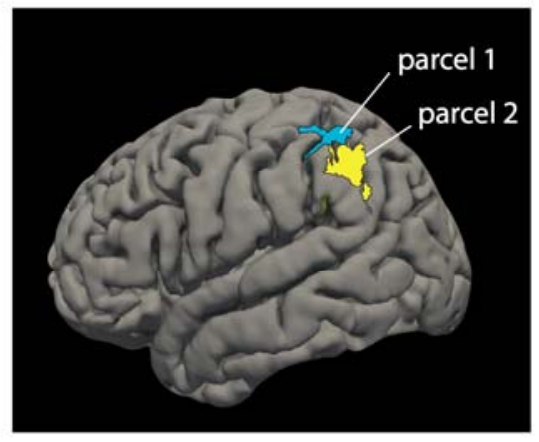

B

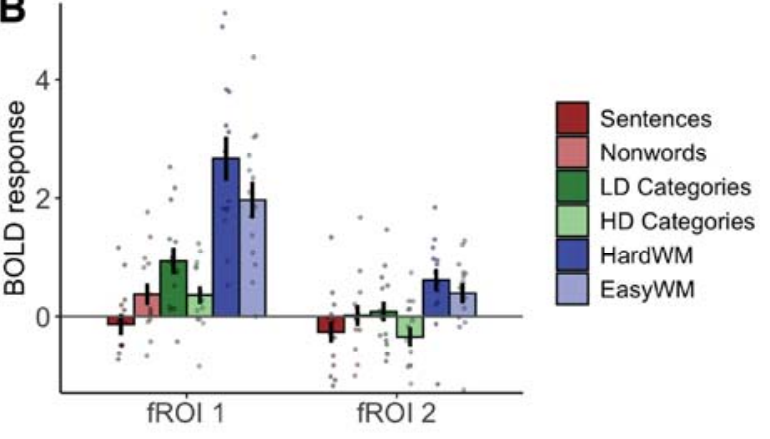

Figure 6. Results of the whole-brain analyses. (A) Parcels defined with the LD>HD categorization contrast. (B) Responses to conditions of interest within the two fROIs (defined as the top $10 \%$ of voxels within each parcel, sorted by the magnitude of the LD>HD response). WM-working memory task.

\subsection{Experiment 3 interim discussion}

In Experiment 3, we used fMRI to examine neural responses to LD and HD categorization. Our main goal was to evaluate the hypothesis that LD categorization relies more heavily on linguistic resources compared to HD categorization. For this purpose, we identified the language network individually in 14 healthy adults and examined its responses during HD and LD categorization. The language network exhibited low responses to both categorization tasks, which did not differ from activations elicited by reading of nonword sequences (a low- 
level control condition). There was no difference between responses to LD categories and responses to $\mathrm{HD}$ categories, contra the prediction that the language network would be selectively or preferentially engaged during LD categorization.

Unlike the language network, the domain-general multiple demand network (also defined individually in each participant) was engaged during categorization, indicating that this task is cognitively challenging. This network responded more strongly to LD than HD categorization, but this effect was small. The whole-brain analyses specifically aimed at identifying regions with stronger responses to LD than HD categorization confirmed these results: both fROIs it identified responded more strongly to a general working memory task than to categorization task, and the LD>HD effect was small or not statistically significant. We conclude that categorization, and LD categorization in particular, relies on domaingeneral multiple demand regions and not on language-specific regions.

Whereas previous work suggested that a region within left angular gyrus is involved in inhibiting irrelevant semantic information (Lewis et al., 2019), as may be required for LD categorization, the results of our study suggest that activation of the language-responsive portion of the left angular gyrus was comparable during LD and HD categorization. If anything, this language fROI showed numerically higher activation during HD categorization, suggesting that it may be recruited for recognizing and thinking about established sets more than for constructing novel sets that may require inhibition of objectirrelevant characteristics. We also did not find significant differences in the engagement of the language fROIs in the left inferior frontal cortex during LD and HD categorization. These results are in contrast to findings from Lupyan et al. (2012), which suggested that TMS to the left inferior frontal cortex disrupted performance on LD but not HD categorization. This may be because the left inferior frontal cortex contains not only language-responsive areas, but also multiple demand areas (Fedorenko et al., 2012; Fedorenko \& Blank, 2020), and interfering with the latter areas' activity may have a disproportionately higher effect on LD categorization.

The response to categorization within the multiple demand network was stronger in the left hemisphere, consistent with the view that label-based categorization recruits the left hemisphere more strongly (e.g., Franklin et al., 2008; Gilbert et al., 2006). This makes the categorization task similar to logic and math, which also evoke left-lateralized responses 
within the multiple demand network (Amalric \& Dehaene, 2016; Monti et al., 2009, 2012; Pinel \& Dehaene, 2009). Importantly, our result demonstrates that, just because the function is left-lateralized, it is not necessarily related to language (contra, e.g., Gilbert et al., 2006; see also Holmes \& Wolff, 2012).

All in all, results from Experiment 3 disconfirm the hypothesis that LD categorization relies on linguistic resources. Instead, they show that categorization recruits the multiple demand brain regions and that $\mathrm{LD}$ categorization is, on average, slightly more effortful that HD categorization.

\section{Discussion}

We reported three experiments that evaluated the hypothesis that linguistic resources are essential for performing feature-based, or low-dimensional (LD), categorization-what we refer to as the 'LD-specific language recruitment hypothesis' (Langland-Hassan et al., 2021; Lupyan, 2009; Lupyan et al., 2012; Lupyan \& Mirman, 2013). In Experiment 1, we aimed to replicate the results of Lupyan and Mirman (2013), who showed a selective impairment in LD categorization in individuals with aphasia. Our results failed to replicate this critical finding, although they did show that naming ability, as measured by Boston Naming Test (BNT) scores, was a significant predictor of overall categorization performance.

In Experiment 2, we modified the experimental design to reduce general task complexity and examined the specific contribution of naming ability to categorization by recruiting a group of participants with low naming scores. Similar to Experiment 1, we found no significant interaction between participant group and LD/HD categorization. However, we did find a significant interaction between BNT and LD/HD categorization for one of the two accuracy measures (hit rate). Although this result lends some support to the LD-specific language recruitment hypothesis, a case-by-case analysis identified an individual with a severe naming impairment (with a score of 1 out of 60 on the BNT) who performed within the neurotypical range on both $\mathrm{HD}$ and $\mathrm{LD}$ categorization. Evidence from patients with brain lesions remains an important way to establish whether specific cognitive capacities are necessary for performance on particular tasks (Rorden \& Karnath, 2004). Such studies have previously demonstrated that many high-order cognitive functions are not affected even in the presence of severe linguistic deficits (e.g., Apperly et al., 2006; Bek et al., 2013; Chen et al., in prep.; 
Varley et al., 2001, 2005; Varley \& Siegal, 2000; Willems et al., 2011). Based on Experiment 2, we therefore concluded that lexical retrieval is not necessary for successful categorization, including categorization based on single features.

In Experiment 3, we used a complementary approach and examined the engagement of the language network and a domain-general multiple-demand network in HD and LD categorization using fMRI in neurotypical adults. The language network was not engaged during either LD or HD categorization: its responses did not significantly differ from responses during the control nonword reading task. This observation goes against the hypothesis that categorization (either LD or HD) relies on linguistic resources. In contrast, the multiple demand network was recruited during the categorization task, consistent with prior evidence of its involvement in diverse cognitively challenging tasks (Assem, Glasser, et al., 2020; Duncan, 2010, 2013; Fedorenko et al., 2013). It responded more strongly during LD than HD categorization, consistent with the hypothesis that LD categorization is more cognitively challenging (e.g., Fedorenko et al., 2011, 2013; Hugdahl et al., 2015; Shashidhara et al., 2019). However, this effect was small and did not come out as statistically significant in any of the individual multiple demand regions in follow-up analyses. In sum, we find little evidence in favor of the LD-specific language recruitment hypothesis.

Why did we find no, little, or inconsistent differences in performance and neural responses between LD and HD categories? A possible explanation is that "LD" and "HD" category types are not natural kinds. As discussed in the introduction, different researchers have emphasized different distinctions among categories, such as natural/ad hoc, taxonomic/thematic, dense/sparse, concrete/abstract, etc. Many of these distinctions are not isomorphic with the $\mathrm{LD} / \mathrm{HD}$ distinction. In particular, $\mathrm{HD}$ categories encompass both taxonomic (e.g., "animals") and thematic (e.g., "non-food things found in the kitchen") categories. Multiple studies show that the processing of taxonomic and thematic relations relies on distinct cognitive and neural mechanisms (e.g., Kalénine et al., 2009; Lewis et al., 2015; Sass et al., 2009; Schwartz et al., 2011; Xu et al., 2018); collapsing them into one "HD" category type leads to substantial within-HD heterogeneity and may therefore obscure potential HD/LD differences.

Furthermore, not all LD categories as defined by Lupyan and Mirman (2013) necessarily involve conceptual processing. For instance, many are based on color, e.g. "things that are 
yellow". Although color is often encoded as part of the conceptual representation of an object, this conceptual representation was not required for the task in question: participants were simply asked to indicate whether the object they were viewing was yellow, and decisions could be made on the basis of surface perceptual features alone. Thus, even if 'true' (semantic) LD categories are indeed harder to process than HD categories, inclusion of perception-based color categories could have prevented us from reliably observing this difference.

Our results appear to be somewhat inconsistent with recent work by Langland-Hassan et al. (2021), who observe that individuals with aphasia were slower when processing abstract categories compared to concrete categories. The authors argue that the abstract/concrete distinction is similar to the $\mathrm{LD} / \mathrm{HD}$ distinction because members of abstract categories share fewer common features. However, another important difference is the kind of features used for categorization. For instance, their example of an abstract category "predict" (which includes a weatherperson and a fortune-teller) relies on an unobservable functional similarity rather than on an observable visual similarity. Unobserved features play an important role in the use of verbal category labels (Gelman \& Roberts, 2017), so it's possible that language mediates categorization based on latent features rather than LD categorization per se. In short, the LD/HD and the abstract/concrete distinction do not cleanly map onto each other, which makes it difficult to compare the results of our experiments to those by LanglandHassan et al. All in all, the typology of category types remains vague and inconsistent, and more careful work should be done to establish meaningful category distinctions and thus facilitate comparisons across studies.

So, what can we conclude about the role of language in categorization, at least in the kind of paradigm introduced by Lupyan \& Mirman (2013)? Based on the current series of studies, we suggest that, in this task, linguistic resources are primarily recruited during the instruction processing stage. In order to successfully sort objects into categories, participants need to read (or listen) and encode the category label, which can explain why participants with severe aphasia may show impairments on this task. Another possible role for language is via verbal rehearsal in order to hold verbal labels active in working memory. Such assistive role of language labels has been observed in conditions of high cognitive demand (e.g., during mathematical calculation; Benn et al., 2012; Klessinger et al., 2012). However, evidence from Experiment 3 shows that language resources implemented in the language network (i.e., 
lexico-semantic and combinatorial processing; Fedorenko et al., 2020) are not involved in the categorization process itself, as evidenced by low activation in the language network during categorization. Instead, categorization in neurotypical individuals might recruit low-level verbal/phonological rehearsal, which appear to rely on lower-level speech processing mechanisms (e.g., Scott \& Perrachione, 2019) and the domain-general multiple-demand network (e.g., Fedorenko et al., 2011; Shashidhara et al., 2020). Thus, the verbal rehearsal account is quite different from L\&M's original LD-specific language recruitment hypothesis.

Other results from psycho- and neurolinguistics also support the view that linguistic resources are not necessary for categorization. If access to linguistic representations were necessary for categorization, categorizing images would take longer than categorizing words; instead, they take approximately the same amount of time (Potter \& Faulconer, 1975). When asked to match a picture with a label, participants do not explicitly generate/rehearse verbal labels in advance unless there is an additional memory demand (e.g., if images disappear from the screen) (Pontillo et al., 2015). Previous work also shows that language is not necessary for performing tasks that require isolating a specific aspect ("feature") of the semantic representation, including theory of mind inferences (Varley et al., 2001; Varley \& Siegal, 2000) and thematic role identification (Ivanova et al., 2021). Our work therefore adds to the growing body of evidence for a separation between linguistic and visual semantic processing.

Overall, our study shows that categorizing items is not a language-dependent task in the adult brain, regardless of whether the categorization is made on the basis of multiple features (HD) or a single feature (LD). Instead, this task relies on domain-general multiple demand neural resources. Our work provides evidence against the view of language as an aid for featurebased (LD) categorization and highlights the value of complementing patient studies with neuroimaging experiments. 


\section{References}

Amalric, M., \& Dehaene, S. (2016). Origins of the brain networks for advanced mathematics in expert mathematicians. Proceedings of the National Academy of Sciences of the United States of America, 113(18), 4909-4917. https://doi.org/10.1073/pnas.1603205113

Amalric, M., \& Dehaene, S. (2019). A distinct cortical network for mathematical knowledge in the human brain. NeuroImage, 189, 19-31. https://doi.org/10.1016/j.neuroimage.2019.01.001

Apperly, I. A., Samson, D., Carroll, N., Hussain, S., \& Humphreys, G. (2006). Intact first-and second-order false belief reasoning in a patient with severely impaired grammar. Social Neuroscience, 1(3-4), 334-348.

Ashby, F. G., Alfonso-Reese, L. A., Turken, A. U., \& Waldron, E. M. (1998). A neuropsychological theory of multiple systems in category learning. Psychological Review, 105(3), 442-481. https://doi.org/10.1037/0033-295x.105.3.442

Ashby, F. G., \& Ell, S. W. (2001). The neurobiology of human category learning. Trends in Cognitive Sciences, 5(5), 204-210. https://doi.org/10.1016/S1364-6613(00)01624-7

Ashby, F. G., \& O’Brien, J. B. (2005). Category learning and multiple memory systems. Trends in Cognitive Sciences, 9(2), 83-89. https://doi.org/10.1016/j.tics.2004.12.003

Assem, M., Blank, I. A., Mineroff, Z., Ademoğlu, A., \& Fedorenko, E. (2020). Activity in the fronto-parietal multiple-demand network is robustly associated with individual differences in working memory and fluid intelligence. Cortex, 131, 1-16. https://doi.org/10.1016/j.cortex.2020.06.013

Assem, M., Glasser, M. F., Van Essen, D. C., \& Duncan, J. (2020). A Domain-General Cognitive Core Defined in Multimodally Parcellated Human Cortex. Cerebral Cortex, 30(8), 4361-4380. https://doi.org/10.1093/cercor/bhaa023 
Baayen, R. H., Davidson, D. J., \& Bates, D. M. (2008). Mixed-effects modeling with crossed random effects for subjects and items. Journal of Memory and Language, 59(4), 390412. https://doi.org/10.1016/j.jml.2007.12.005

Bain, A. (1864). The senses and the intellect. Longman, Green, Longman, Roberts, and Green.

Baldo, J. V., Bunge, S. A., Wilson, S. M., \& Dronkers, N. F. (2010). Is relational reasoning dependent on language? A voxel-based lesion symptom mapping study. Brain and Language, 113(2), 59-64. https://doi.org/10.1016/j.bandl.2010.01.004

Baldo, J. V., Paulraj, S. R., Curran, B. C., \& Dronkers, N. F. (2015). Impaired reasoning and problem-solving in individuals with language impairment due to aphasia or language delay. Frontiers in Psychology, 6, 1523. https://doi.org/10.3389/fpsyg.2015.01523

Barsalou, L. W. (1983). Ad hoc categories. Memory \& Cognition, 11(3), 211-227. https://doi.org/10.3758/BF03196968

Bates, D., Mächler, M., Bolker, B., \& Walker, S. (2015). Fitting Linear Mixed-Effects Models Using lme4. Journal of Statistical Software, 67(1), 1-48. https://doi.org/10.18637/jss.v067.i01

Bates, E., Wilson, S. M., Saygin, A. P., Dick, F., Sereno, M. I., Knight, R. T., \& Dronkers, N. F. (2003). Voxel-based lesion-symptom mapping. Nature Neuroscience, 6(5), 448450. https://doi.org/10.1038/nn1050

Bek, J., Blades, M., Siegal, M., \& Varley, R. (2013). Dual-Task Interference in Spatial Reorientation: Linguistic and Nonlinguistic Factors. Spatial Cognition \& Computation, 13(1), 26-49. https://doi.org/10.1080/13875868.2011.590622

Benjamini, Y., \& Hochberg, Y. (1995). Controlling the False Discovery Rate: A Practical and Powerful Approach to Multiple Testing. Journal of the Royal Statistical Society. Series B (Methodological), 57(1), 289-300. JSTOR. 
Benn, Y., Wilkinson, I. D., Zheng, Y., Kadosh, K. C., Romanowski, C. A. J., Siegal, M., \& Varley, R. (2013). Differentiating core and co-opted mechanisms in calculation: The neuroimaging of calculation in aphasia. Brain and Cognition, 82(3), 254-264. https://doi.org/10.1016/j.bandc.2013.04.012

Benn, Y., Zheng, Y., Wilkinson, I. D., Siegal, M., \& Varley, R. (2012). Language in calculation: A core mechanism? Neuropsychologia, 50(1), 1-10. https://doi.org/10.1016/j.neuropsychologia.2011.09.045

Bermúdez, J. L. (2007). Thinking without words. Oxford University Press.

Bickerton, D. (1995). Language and Human Behavior. University of Washington Press.

Blank, I. A., Kanwisher, N., \& Fedorenko, E. (2014). A functional dissociation between language and multiple-demand systems revealed in patterns of BOLD signal fluctuations. Journal of Neurophysiology, 112(5), 1105-1118. https://doi.org/10.1152/jn.00884.2013

Braga, R. M., DiNicola, L. M., Becker, H. C., \& Buckner, R. L. (2020). Situating the LeftLateralized Language Network in the Broader Organization of Multiple Specialized Large-Scale Distributed Networks. Journal of Neurophysiology. https://doi.org/10.1152/jn.00753.2019

Brojde, C. L., Porter, C., \& Colunga, E. (2011). Words can slow down category learning. Psychonomic Bulletin \& Review, 18(4), 798-804. https://doi.org/10.3758/s13423-0110103-Z

Burger, R. A., \& Muma, J. R. (1980). Cognitive distancing in mediated categorization in aphasia. Journal of Psycholinguistic Research, 9(4), 355-365.

https://doi.org/10.1007/BF01067448 
Caramazza, A., Berndt, R. S., \& Brownell, H. H. (1982). The semantic deficit hypothesis:

Perceptual parsing and object classification by aphasic patients. Brain and Language,

15(1), 161-189. https://doi.org/10.1016/0093-934x(82)90054-2

Caramazza, A., \& Coltheart, M. (2006). Cognitive Neuropsychology twenty years on.

Cognitive Neuropsychology, 23(1), 3-12.

https://doi.org/10.1080/02643290500443250

Carruthers, P. (2002). The cognitive functions of language. The Behavioral and Brain

Sciences, 25(6), 657-674; discussion 674-725.

https://doi.org/10.1017/s0140525x02000122

Chen, X., Affourtit, J., Norman-Haignere, S., Jouravlev, O., Malik-Moraleda, S., Kean, H.

H., Regev, T., McDermott, J. H., \& Fedorenko, E. (in prep.). The fronto-temporal language system does not support the processing of music.

Cohen, R., Kelter, S., \& Woll, G. (1980). Analytical competence and language impairment in aphasia. Brain and Language, 10(2), 331-347. https://doi.org/10.1016/0093934X(80)90060-7

Cohen, R., \& Woll, G. (1981). Facets of Analytical Processing in Aphasia: A Picture Ordering Task. Cortex, 17(4), 557-569. https://doi.org/10.1016/S00109452(81)80062-7

Couchman, J. J., Coutinho, M. V. C., \& Smith, J. D. (2010). Rules and Resemblance: Their Changing Balance in the Category Learning of Humans (Homo sapiens) and Monkeys (Macaca mulatta). Journal of Experimental Psychology. Animal Behavior Processes, 36(2), 172-183. https://doi.org/10.1037/a0016748

Darwin, C. (1871). The descent of man and selection in relation to sex. John Murray. 
Davidoff, J., \& Roberson, D. (2004). Preserved thematic and impaired taxonomic categorisation: A case study. Language and Cognitive Processes, 19(1), 137-174. https://doi.org/10.1080/01690960344000125

De Renzi, E., \& Spinnler, H. (1967). Impaired Performance on Color Tasks in Patients with Hemispheric Damage. Cortex, 3(2), 194-217. https://doi.org/10.1016/S00109452(67)80012-1

Dennett, D. C. (1994). The role of language in intelligence. In What is Intelligence? The Darwin College Lectures, ed. Jean Khalfa, Cambridge University Press.

Duncan, J. (2010). The multiple-demand (MD) system of the primate brain: Mental programs for intelligent behaviour. Trends in Cognitive Sciences, 14(4), 172-179. https://doi.org/10.1016/j.tics.2010.01.004

Duncan, J. (2013). The Structure of Cognition: Attentional Episodes in Mind and Brain. Neuron, 80(1), 35-50. https://doi.org/10.1016/j.neuron.2013.09.015

Duncan, J., \& Owen, A. M. (2000). Common regions of the human frontal lobe recruited by diverse cognitive demands. Trends in Neurosciences, 23(10), 475-483. https://doi.org/10.1016/S0166-2236(00)01633-7

Elwert, F., \& Winship, C. (2014). Endogenous Selection Bias: The Problem of Conditioning on a Collider Variable. Annual Review of Sociology, 40(1), 31-53. https://doi.org/10.1146/annurev-soc-071913-043455

Fedorenko, E., Behr, M. K., \& Kanwisher, N. (2011). Functional specificity for high-level linguistic processing in the human brain. Proceedings of the National Academy of Sciences, 108(39), 16428-16433. https://doi.org/10.1073/pnas.1112937108

Fedorenko, E., \& Blank, I. A. (2020). Broca's Area Is Not a Natural Kind. Trends in Cognitive Sciences. https://doi.org/10.1016/j.tics.2020.01.001 
Fedorenko, E., Blank, I. A., Siegelman, M., \& Mineroff, Z. (2020). Lack of selectivity for syntax relative to word meanings throughout the language network. Cognition, 203, 104348. https://doi.org/10.1016/j.cognition.2020.104348

Fedorenko, E., Duncan, J., \& Kanwisher, N. (2012). Language-Selective and DomainGeneral Regions Lie Side by Side within Broca's Area. Current Biology, 22(21), 2059-2062. https://doi.org/10.1016/j.cub.2012.09.011

Fedorenko, E., Duncan, J., \& Kanwisher, N. (2013). Broad domain generality in focal regions of frontal and parietal cortex. Proceedings of the National Academy of Sciences of the United States of America, 110(41), 16616-16621.

https://doi.org/10.1073/pnas.1315235110

Fedorenko, E., Hsieh, P.-J., Nieto-Castañón, A., Whitfield-Gabrieli, S., \& Kanwisher, N. (2010). New method for fMRI investigations of language: Defining ROIs functionally in individual subjects. Journal of Neurophysiology, 104(2), 1177-1194. https://doi.org/10.1152/jn.00032.2010

Ferguson, B., \& Waxman, S. (2017). Linking language and categorization in infancy. Journal of Child Language, 44(3), 527-552. https://doi.org/10.1017/S0305000916000568

Franklin, A., Drivonikou, G. V., Clifford, A., Kay, P., Regier, T., \& Davies, I. R. L. (2008). Lateralization of categorical perception of color changes with color term acquisition. Proceedings of the National Academy of Sciences, 105(47), 18221-18225. https://doi.org/10.1073/pnas.0809952105

Friston, K. J., Holmes, A. P., Worsley, K. J., Poline, J.-P., Frith, C. D., \& Frackowiak, R. S. J. (1994). Statistical parametric maps in functional imaging: A general linear approach. Human Brain Mapping, 2(4), 189-210. https://doi.org/10.1002/hbm.460020402 
Gainotti, G., D’Erme, P., Villa, G., \& Caltagirone, C. (1986). Focal brain lesions and intelligence: A study with a new version of Raven's Colored Matrices. Journal of Clinical and Experimental Neuropsychology, 8(1), 37-50.

https://doi.org/10.1080/01688638608401295

Gelman, S. A., \& Roberts, S. O. (2017). How language shapes the cultural inheritance of categories. Proceedings of the National Academy of Sciences, 114(30), 7900-7907. https://doi.org/10.1073/pnas.1621073114

Gershkoff-Stowe, L., Thal, D. J., Smith, L. B., \& Namy, L. L. (1997). Categorization and Its Developmental Relation to Early Language. Child Development, 68(5), 843-859. https://doi.org/10.2307/1132037

Gilbert, A. L., Regier, T., Kay, P., \& Ivry, R. B. (2006). Whorf hypothesis is supported in the right visual field but not the left. Proceedings of the National Academy of Sciences of the United States of America, 103(2), 489-494. https://doi.org/10.1073/pnas.0509868103

Goodglass, H., Kaplan, E., \& Weintraub, S. (1983). Boston naming test. Lea \& Febiger Philadelphia, PA.

Hjelmquist, E. K. (1989). Concept formation in non-verbal categorization tasks in braindamaged patients with and without aphasia. Scandinavian Journal of Psychology, 30(4), 243-254. https://doi.org/10.1111/j.1467-9450.1989.tb01087.x

Holmes, K. J., \& Wolff, P. (2012). Does categorical perception in the left hemisphere depend on language? Journal of Experimental Psychology. General, 141(3), 439-443. https://doi.org/10.1037/a0027289

Hough, M. S. (1993). Categorization in aphasia: Access and organization of goal-derived and common categories. Aphasiology, 7(4), 335-357. https://doi.org/10.1080/02687039308249515 
Hugdahl, K., Raichle, M. E., Mitra, A., \& Specht, K. (2015). On the existence of a generalized non-specific task-dependent network. Frontiers in Human Neuroscience, 9. https://doi.org/10.3389/fnhum.2015.00430

IBM Corp. (2013). IBM SPSS Statistics for Windows (22.0) [Computer software]. https://hadoop.apache.org

Ioannidis, J. P. A. (2014). How to Make More Published Research True. PLOS Medicine, 11(10), e1001747. https://doi.org/10.1371/journal.pmed.1001747

Ivanova, A. A., Mineroff, Z., Zimmerer, V., Kanwisher, N., Varley, R., \& Fedorenko, E. (2021). The Language Network is Recruited but Not Required for Nonverbal Event Semantics. Neurobiology of Language, 1-26. https://doi.org/10.1162/nol_a_00030

Jaeger, T. F. (2008). Categorical Data Analysis: Away from ANOVAs (transformation or not) and towards Logit Mixed Models. Journal of Memory and Language, 59(4), 434-446. https://doi.org/10.1016/j.jml.2007.11.007

Julian, J. B., Fedorenko, E., Webster, J., \& Kanwisher, N. (2012). An algorithmic method for functionally defining regions of interest in the ventral visual pathway. NeuroImage, 60(4), 2357-2364. https://doi.org/10.1016/j.neuroimage.2012.02.055

Kalénine, S., Peyrin, C., Pichat, C., Segebarth, C., Bonthoux, F., \& Baciu, M. (2009). The sensory-motor specificity of taxonomic and thematic conceptual relations: A behavioral and fMRI study. NeuroImage, 44(3), 1152-1162. https://doi.org/10.1016/j.neuroimage.2008.09.043

Kemler Nelson, D. G. (1984). The effect of intention on what concepts are acquired. Journal of Verbal Learning and Verbal Behavior, 23(6), 734-759.

https://doi.org/10.1016/S0022-5371(84)90442-0 
Kim, H. S. (2002). We talk, therefore we think? A cultural analysis of the effect of talking on thinking. Journal of Personality and Social Psychology, 83(4), 828-842. https://doi.org/10.1037/0022-3514.83.4.828

Klessinger, N., Szczerbinski, M., \& Varley, R. (2012). The role of number words: The phonological length effect in multidigit addition. Memory \& Cognition, 40(8), 12891302. https://doi.org/10.3758/s13421-012-0228-y

Kloos, H., \& Sloutsky, V. M. (2008). What's behind different kinds of kinds: Effects of statistical density on learning and representation of categories. Journal of Experimental Psychology: General, 137(1), 52-72. https://doi.org/10.1037/00963445.137 .1 .52

Koemeda-Lutz, M., Cohen, R., \& Meier, E. (1987). Organization of and access to semantic memory in aphasia. Brain and Language, 30(2), 321-337. https://doi.org/10.1016/0093-934X(87)90106-4

Kriegeskorte, N., Simmons, W. K., Bellgowan, P. S. F., \& Baker, C. I. (2009). Circular analysis in systems neuroscience: The dangers of double dipping. Nature Neuroscience, 12(5), 535-540. https://doi.org/10.1038/nn.2303

Kuznetsova, A., Brockhoff, P. B., \& Christensen, R. H. B. (2017). 1merTest Package: Tests in Linear Mixed Effects Models. Journal of Statistical Software, 82(1), 1-26. https://doi.org/10.18637/jss.v082.i13

Langland-Hassan, P., Faries, F. R., Gatyas, M., Dietz, A., \& Richardson, M. J. (2021). Assessing abstract thought and its relation to language with a new nonverbal paradigm: Evidence from aphasia. Cognition, 211, 104622. https://doi.org/10.1016/j.cognition.2021.104622 
Le Dorze, G., \& Nespoulous, J. L. (1989). Anomia in moderate aphasia: Problems in accessing the lexical representation. Brain and Language, 37(3), 381-400. https://doi.org/10.1016/0093-934x(89)90026-6

Lewis, G. A., Poeppel, D., \& Murphy, G. L. (2015). The neural bases of taxonomic and thematic conceptual relations: An MEG study. Neuropsychologia. https://doi.org/10.1016/j.neuropsychologia.2015.01.011

Lewis, G. A., Poeppel, D., \& Murphy, G. L. (2019). Contrasting Semantic versus Inhibitory Processing in the Angular Gyrus: An fMRI Study. Cerebral Cortex, 29(6), 24702481. https://doi.org/10.1093/cercor/bhy118

Lupyan, G. (2009). Extracommunicative functions of language: Verbal interference causes selective categorization impairments. Psychonomic Bulletin \& Review, 16(4), 711718. https://doi.org/10.3758/PBR.16.4.711

Lupyan, G. (2012). Linguistically Modulated Perception and Cognition: The Label-Feedback Hypothesis. Frontiers in Psychology, 3. https://doi.org/10.3389/fpsyg.2012.00054

Lupyan, G., \& Casasanto, D. (2015). Meaningless words promote meaningful categorization. Language and Cognition: An Interdisciplinary Journal of Language and Cognitive Science, 7(2), 167-193. https://doi.org/10.1017/langcog.2014.21

Lupyan, G., \& Mirman, D. (2013). Linking language and categorization: Evidence from aphasia. Cortex, 49(5), 1187-1194. https://doi.org/10.1016/j.cortex.2012.06.006

Lupyan, G., Mirman, D., Hamilton, R., \& Thompson-Schill, S. L. (2012). Categorization is modulated by transcranial direct current stimulation over left prefrontal cortex. Cognition, 124(1), 36-49. https://doi.org/10.1016/j.cognition.2012.04.002

Lupyan, G., Rakison, D. H., \& McClelland, J. L. (2007). Language is not Just for Talking: Redundant Labels Facilitate Learning of Novel Categories. Psychological Science, 18(12), 1077-1083. https://doi.org/10.1111/j.1467-9280.2007.02028.x 
Mahowald, K., \& Fedorenko, E. (2016). Reliable individual-level neural markers of highlevel language processing: A necessary precursor for relating neural variability to behavioral and genetic variability. NeuroImage, 139, 74-93.

https://doi.org/10.1016/j.neuroimage.2016.05.073

Mareschal, D., \& Quinn, P. C. (2001). Categorization in infancy. Trends in Cognitive Sciences, 5(10), 443-450. https://doi.org/10.1016/S1364-6613(00)01752-6

Mervis, C. B., \& Rosch, E. (1981). Categorization of Natural Objects. Annual Review of Psychology, 32(1), 89-115. https://doi.org/10.1146/annurev.ps.32.020181.000513

Mineroff, Z., Blank, I. A., Mahowald, K., \& Fedorenko, E. (2018). A robust dissociation among the language, multiple demand, and default mode networks: Evidence from inter-region correlations in effect size. Neuropsychologia, 119, 501-511. https://doi.org/10.1016/j.neuropsychologia.2018.09.011

Mirman, D., Landrigan, J.-F., \& Britt, A. E. (2017). Taxonomic and Thematic Semantic Systems. Psychological Bulletin, 143(5), 499-520. https://doi.org/10.1037/bu10000092

Monti, M. M., Parsons, L. M., \& Osherson, D. N. (2009). The boundaries of language and thought in deductive inference. Proceedings of the National Academy of Sciences, 106(30), 12554-12559. https://doi.org/10.1073/pnas.0902422106

Monti, M. M., Parsons, L. M., \& Osherson, D. N. (2012). Thought beyond language: Neural dissociation of algebra and natural language. Psychological Science, 23(8), 914-922. https://doi.org/10.1177/0956797612437427

Murphy, G. (2002). The big book of concepts. MIT Press.

Nieto-Castañón, A., \& Fedorenko, E. (2012). Subject-specific functional localizers increase sensitivity and functional resolution of multi-subject analyses. NeuroImage, 63(3), 1646-1669. https://doi.org/10.1016/j.neuroimage.2012.06.065 
Pearce, J. M. (1994). Discrimination and Categorization. In N. J. Mackintosh (Ed.), Animal Learning and Cognition (pp. 109-134). Academic Press. https://doi.org/10.1016/B978-0-08-057169-0.50011-5

Perry, L. K., \& Lupyan, G. (2014). The role of language in multi-dimensional categorization: Evidence from transcranial direct current stimulation and exposure to verbal labels. Brain and Language, 135, 66-72. https://doi.org/10.1016/j.bandl.2014.05.005

Petersen, S. E., \& Posner, M. I. (2012). The Attention System of the Human Brain: 20 Years After. Annual Review of Neuroscience, 35, 73-89. https://doi.org/10.1146/annurevneuro-062111-150525

Pinel, P., \& Dehaene, S. (2009). Beyond Hemispheric Dominance: Brain Regions Underlying the Joint Lateralization of Language and Arithmetic to the Left Hemisphere. Journal of Cognitive Neuroscience, 22(1), 48-66. https://doi.org/10.1162/jocn.2009.21184

Plunkett, K., Hu, J.-F., \& Cohen, L. B. (2008). Labels can override perceptual categories in early infancy. Cognition, 106(2), 665-681. https://doi.org/10.1016/j.cognition.2007.04.003

Poldrack, R. A., Baker, C. I., Durnez, J., Gorgolewski, K. J., Matthews, P. M., Munafò, M. R., Nichols, T. E., Poline, J.-B., Vul, E., \& Yarkoni, T. (2017). Scanning the horizon: Towards transparent and reproducible neuroimaging research. Nature Reviews Neuroscience, 18(2), 115-126. https://doi.org/10.1038/nrn.2016.167

Pontillo, D. F., Salverda, A. P., \& Tanenhaus, M. K. (2015). Flexible Use of Phonological and Visual Memory in Language-mediated Visual Search. Proceedings of the 37th Meeting of the Cognitive Science Society.

Posner, M. I., \& Petersen, S. E. (1990). The attention system of the human brain. Annual Review of Neuroscience, 13, 25-42. https://doi.org/10.1146/annurev.ne.13.030190.000325 
Potter, M. C., \& Faulconer, B. A. (1975). Time to understand pictures and words. Nature, 253(5491), 437-438. https://doi.org/10.1038/253437a0

Rabe, M. M., Vasishth, S., Hohenstein, S., Kliegl, R., \& Schad, D. J. (2020). hypr: An R package for hypothesis-driven contrast coding. Journal of Open Source Software, 5(48), 2134. https://doi.org/10.21105/joss.02134

Rorden, C., \& Karnath, H.-O. (2004). Using human brain lesions to infer function: A relic from a past era in the fMRI age? Nature Reviews Neuroscience, 5(10), 812-819. https://doi.org/10.1038/nrn1521

Sachs, O., Weis, S., Zellagui, N., Huber, W., Zvyagintsev, M., Mathiak, K., \& Kircher, T. (2008). Automatic processing of semantic relations in fMRI: Neural activation during semantic priming of taxonomic and thematic categories. Brain Research, 1218, 194205. https://doi.org/10.1016/j.brainres.2008.03.045

Sass, K., Sachs, O., Krach, S., \& Kircher, T. (2009). Taxonomic and thematic categories: Neural correlates of categorization in an auditory-to-visual priming task using fMRI. Brain Research, 1270, 78-87. https://doi.org/10.1016/j.brainres.2009.03.013

Saxe, R., Brett, M., \& Kanwisher, N. (2006). Divide and conquer: A defense of functional localizers. NeuroImage, 30(4), 1088-1096; discussion 1097-1099. https://doi.org/10.1016/j.neuroimage.2005.12.062

Schwartz, M. F., Kimberg, D. Y., Walker, G. M., Brecher, A., Faseyitan, O. K., Dell, G. S., Mirman, D., \& Coslett, H. B. (2011). Neuroanatomical dissociation for taxonomic and thematic knowledge in the human brain. Proceedings of the National Academy of Sciences of the United States of America, 108(20), 8520-8524.

https://doi.org/10.1073/pnas.1014935108 
Scott, T. L., Gallée, J., \& Fedorenko, E. (2017). A new fun and robust version of an fMRI localizer for the frontotemporal language system. Cognitive Neuroscience, 8(3), 167176. https://doi.org/10.1080/17588928.2016.1201466

Scott, T. L., \& Perrachione, T. K. (2019). Common cortical architectures for phonological working memory identified in individual brains. NeuroImage, 202, 116096. https://doi.org/10.1016/j.neuroimage.2019.116096

Shashidhara, S., Mitchell, D. J., Erez, Y., \& Duncan, J. (2019). Progressive Recruitment of the Frontoparietal Multiple-demand System with Increased Task Complexity, Time Pressure, and Reward. Journal of Cognitive Neuroscience, 31(11), 1617-1630. https://doi.org/10.1162/jocn_a_01440

Shashidhara, S., Spronkers, F. S., \& Erez, Y. (2020). Individual-subject Functional Localization Increases Univariate Activation but Not Multivariate Pattern Discriminability in the "Multiple-demand" Frontoparietal Network. Journal of Cognitive Neuroscience, 32(7), 1348-1368. https://doi.org/10.1162/jocn_a_01554

Siegal, M., \& Varley, R. (2006). Aphasia, language, and theory of mind. Social Neuroscience, 1(3-4), 167-174. https://doi.org/10.1080/17470910600985597

Sloutsky, V. M. (2010). From Perceptual Categories to Concepts: What Develops? Cognitive Science, 34(7), 1244-1286. https://doi.org/10.1111/j.1551-6709.2010.01129.x

Sloutsky, V. M., \& Fisher, A. V. (2004). Induction and categorization in young children: A similarity-based model. Journal of Experimental Psychology. General, 133(2), 166188. https://doi.org/10.1037/0096-3445.133.2.166

Smith, E. E., \& Medin, D. L. (1981). Categories and concepts. Harvard University Press.

Smith, L. B., \& Heise, D. (1992). Perceptual Similarity and Conceptual Structure. In B. Burns (Ed.), Advances in Psychology (Vol. 93, pp. 233-272). North-Holland. https://doi.org/10.1016/S0166-4115(08)61009-2 
Vallila-Rohter, S., \& Kiran, S. (2015). An Examination of Strategy Implementation During Abstract Nonlinguistic Category Learning in Aphasia. Journal of Speech, Language, and Hearing Research: JSLHR, 58(4), 1195-1209.

https://doi.org/10.1044/2015_JSLHR-L-14-0257

Varley, R. A., Klessinger, N. J. C., Romanowski, C. A. J., \& Siegal, M. (2005). Agrammatic but numerate. Proceedings of the National Academy of Sciences of the United States of America, 102(9), 3519-3524. https://doi.org/10.1073/pnas.0407470102

Varley, R. A., \& Siegal, M. (2000). Evidence for cognition without grammar from causal reasoning and "theory of mind" in an agrammatic aphasic patient. Current Biology: $C B, 10(12), 723-726$. https://doi.org/10.1016/s0960-9822(00)00538-8

Varley, R. A., Siegal, M., \& Want, S. C. (2001). Severe impairment in grammar does not preclude theory of mind. Neurocase, 7(6), 489-493. https://doi.org/10.1093/neucas/7.6.489

Wasserman, E., Kiedinger, R. E., \& Bhatt, R. (1988). Conceptual behavior in pigeons: Categories, subcategories, and pseudocategories. https://doi.org/10.1037/00977403.14.3.235

Waxman, S. R., \& Gelman, S. A. (2009). Early word-learning entails reference, not merely associations. Trends in Cognitive Sciences, 13(6), 258-263. https://doi.org/10.1016/j.tics.2009.03.006

Whitehouse, P., Caramazza, A., \& Zurif, E. (1978). Naming in aphasia: Interacting effects of form and function. Brain and Language, 6(1), 63-74. https://doi.org/10.1016/0093$934 \times(78) 90044-5$

Willems, R. M., Benn, Y., Hagoort, P., Toni, I., \& Varley, R. (2011). Communicating without a functioning language system: Implications for the role of language in 
mentalizing. Neuropsychologia, 49(11), 3130-3135.

https://doi.org/10.1016/j.neuropsychologia.2011.07.023

Willems, R. M., der Haegen, L. V., Fisher, S. E., \& Francks, C. (2014). On the other hand: Including left-handers in cognitive neuroscience and neurogenetics. Nature Reviews Neuroscience, 15(3), 193-201. https://doi.org/10.1038/nrn3679

Xu, Y., Wang, X., Wang, X., Men, W., Gao, J.-H., \& Bi, Y. (2018). Doctor, Teacher, and Stethoscope: Neural Representation of Different Types of Semantic Relations. The Journal of Neuroscience, 38(13), 3303-3317.

https://doi.org/10.1523/JNEUROSCI.2562-17.2018 


\section{Appendix 1: Experiment 3, Material Presentation Details}

Table 1. Distribution of category use across participants (i.e., the number of times each participant saw each category during the categorization experiment, summed across runs).

\begin{tabular}{|c|c|c|c|c|c|c|c|c|c|c|c|c|c|c|c|}
\hline & \multicolumn{14}{|c|}{ Subject ID } \\
\hline Condition & Category & 1 & 2 & 3 & 4 & 5 & 6 & 7 & 8 & 9 & 10 & 11 & 12 & 13 & 14 \\
\hline HD & animals that live in water & 1 & 1 & 1 & 0 & 0 & 0 & 1 & 1 & 2 & 1 & 1 & 2 & 2 & 2 \\
\hline HD & birds & 2 & 0 & 1 & 1 & 1 & 3 & 1 & 2 & 1 & 2 & 3 & 1 & 3 & 1 \\
\hline HD & clothes & 1 & 1 & 1 & 1 & 1 & 0 & 1 & 0 & 0 & 2 & 1 & 0 & 1 & 1 \\
\hline HD & dangerous animals & 2 & 1 & 2 & 1 & 1 & 0 & 1 & 1 & 1 & 1 & 1 & 0 & 0 & 1 \\
\hline HD & farm animals & 1 & 1 & 3 & 2 & 0 & 0 & 2 & 2 & 2 & 1 & 3 & 1 & 2 & 1 \\
\hline HD & fruit & 1 & 2 & 1 & 1 & 2 & 2 & 0 & 0 & 1 & 1 & 1 & 2 & 1 & 0 \\
\hline HD & home appliances & 2 & 2 & 0 & 1 & 1 & 2 & 2 & 1 & 0 & 1 & 0 & 2 & 0 & 1 \\
\hline HD & insects & 2 & 2 & 1 & 3 & 1 & 2 & 1 & 2 & 1 & 2 & 1 & 2 & 1 & 2 \\
\hline HD & musical instruments & 0 & 1 & 1 & 0 & 3 & 0 & 2 & 1 & 2 & 2 & 2 & 1 & 1 & 1 \\
\hline HD & non food things found in the kitchen & 2 & 1 & 0 & 0 & 2 & 2 & 0 & 2 & 2 & 1 & 1 & 2 & 0 & 1 \\
\hline HD & objects found in the laundry room & 1 & 1 & 0 & 2 & 1 & 1 & 1 & 2 & 1 & 1 & 1 & 0 & 1 & 1 \\
\hline HD & objects that hold water & 0 & 1 & 2 & 2 & 0 & 1 & 2 & 0 & 0 & 1 & 1 & 1 & 2 & 2 \\
\hline HD & objects used for transportation & 1 & 2 & 1 & 1 & 1 & 1 & 0 & 1 & 3 & 1 & 0 & 0 & 1 & 1 \\
\hline HD & things that fly & 1 & 2 & 3 & 1 & 0 & 1 & 2 & 2 & 1 & 0 & 1 & 2 & 0 & 2 \\
\hline HD & tools & 1 & 0 & 0 & 2 & 2 & 2 & 2 & 0 & 0 & 0 & 0 & 1 & 0 & 0 \\
\hline HD & vegetables & 0 & 0 & 1 & 0 & 2 & 1 & 0 & 1 & 1 & 1 & 1 & 1 & 3 & 1 \\
\hline LD & animals with stripes & 2 & 1 & 2 & 2 & 0 & 1 & 2 & 0 & 1 & 2 & 0 & 1 & 0 & 2 \\
\hline LD & long thin objects & 0 & 0 & 2 & 2 & 1 & 1 & 1 & 1 & 0 & 2 & 2 & 1 & 2 & 2 \\
\hline LD & small objects & 1 & 2 & 0 & 1 & 2 & 1 & 0 & 1 & 1 & 1 & 1 & 0 & 1 & 1 \\
\hline LD & things made of wood & 1 & 1 & 1 & 3 & 2 & 1 & 2 & 1 & 0 & 1 & 2 & 2 & 0 & 0 \\
\hline LD & things that are blue & 2 & 1 & 1 & 1 & 1 & 1 & 1 & 1 & 0 & 0 & 1 & 0 & 1 & 1 \\
\hline LD & things that are brown & 1 & 2 & 1 & 0 & 3 & 1 & 2 & 2 & 2 & 1 & 2 & 0 & 1 & 1 \\
\hline LD & things that are green & 1 & 0 & 1 & 1 & 0 & 2 & 1 & 1 & 2 & 2 & 1 & 1 & 0 & 1 \\
\hline LD & things that are orange & 2 & 1 & 2 & 2 & 1 & 2 & 0 & 1 & 1 & 1 & 0 & 2 & 2 & 1 \\
\hline LD & things that are red & 1 & 1 & 1 & 1 & 0 & 1 & 1 & 1 & 2 & 0 & 2 & 1 & 2 & 1 \\
\hline LD & things that are round & 1 & 1 & 1 & 1 & 1 & 1 & 0 & 1 & 3 & 1 & 2 & 0 & 1 & 1 \\
\hline LD & things that are soft & 0 & 0 & 2 & 0 & 2 & 1 & 1 & 2 & 1 & 0 & 2 & 2 & 1 & 2 \\
\hline LD & things that are very large & 1 & 1 & 1 & 2 & 1 & 2 & 1 & 1 & 1 & 1 & 1 & 2 & 3 & 0 \\
\hline LD & things that are white & 3 & 2 & 1 & 1 & 1 & 0 & 1 & 0 & 1 & 1 & 0 & 3 & 0 & 2 \\
\hline LD & things that are yellow & 2 & 3 & 0 & 0 & 0 & 1 & 2 & 2 & 1 & 3 & 1 & 1 & 2 & 0 \\
\hline LD & things with doors & 0 & 1 & 1 & 0 & 1 & 1 & 2 & 2 & 1 & 1 & 0 & 1 & 2 & 3 \\
\hline LD & things with handles & 0 & 1 & 1 & 1 & 2 & 1 & 1 & 1 & 1 & 1 & 1 & 1 & 0 & 0 \\
\hline
\end{tabular}




\section{Appendix 2: Experiment 3, fROI-Specific Results}

Condition contrasts were designed to test the following null hypotheses.

Language network:
(1) $\frac{H D+L D}{2}=0$
(2) $L D=H D$ (main)
(3) $\frac{H D+L D 2}{2}=$ Sentences
(4) $\frac{H D+L D}{2}=$ Nonwords

LD - low-dimensional categorization; HD - high-dimensional categorization.

Multiple demand network:
(1) $\frac{H D+L D}{2}=0$
(2) $L D=H D \quad$ (main)
(3) $\operatorname{HardWM}=$ EasyWM
(4) $\frac{H D+L D}{2}=\frac{H \operatorname{Hard} W M+E a s y W M}{2}$
(5) $\frac{H D+L D}{2}=$ Sentences
(6) $\frac{H D+L D}{2}=$ Nonwords

HardWM - hard working memory task, EasyWM - easy working memory task.

Putative LD categorization regions (results reported in the main text):
(1) $\frac{H D+L D}{2}=0$
(2) $L D=H D \quad$ (main)
(3) $\operatorname{HardWM}=$ EasyWM
(4) Sentences $=$ Nonwords
(5) $\frac{H D+L D}{2}=\frac{H \operatorname{ard} W M+E \text { Easy } W M}{2}$
(6) $\frac{H D+L D}{2}=$ Nonwords 
Table 1. Mixed-effect linear regression results for language fROIs. p-values were FDR-corrected for the number of fROIs. Significant p-values are highlighted in bold. $S$ - sentence reading, $N$ - nonword reading, $L D$ - low-dimensional categorization, $H D$ - high-dimensional categorization.

\begin{tabular}{|c|c|c|c|}
\hline ROI & Regression Term & Beta & $p$-value \\
\hline \multirow[t]{4}{*}{ IFGorb } & Categorization>0 & 0.48 & .005 \\
\hline & $\mathrm{LD}>\mathrm{HD}$ & 0.04 & .932 \\
\hline & S>Categorization & 1.21 & $<.001$ \\
\hline & $\mathrm{N}>$ Categorization & 0.02 & .905 \\
\hline \multirow[t]{4}{*}{ IFG } & Categorization >0 & 0.90 & $<.001$ \\
\hline & $\mathrm{LD}>\mathrm{HD}$ & 0.02 & .932 \\
\hline & S>Categorization & 1.41 & $<.001$ \\
\hline & $\mathrm{N}>$ Categorization & -0.15 & .510 \\
\hline \multirow[t]{4}{*}{ MFG } & Categorization $>0$ & 0.63 & .005 \\
\hline & $\mathrm{LD}>\mathrm{HD}$ & 0.11 & .932 \\
\hline & S>Categorization & 2.41 & $<.001$ \\
\hline & $\mathbf{N}>$ Categorization & 0.75 & .031 \\
\hline \multirow[t]{4}{*}{ PostTemp } & Categorization $>0$ & 0.27 & .094 \\
\hline & $\mathrm{LD}>\mathrm{HD}$ & 0.10 & .932 \\
\hline & S>Categorization & 1.81 & $<.001$ \\
\hline & $\mathrm{N}>$ Categorization & 0.26 & .114 \\
\hline \multirow[t]{4}{*}{ AntTemp } & Categorization $>0$ & -0.02 & .760 \\
\hline & $\mathrm{LD}>\mathrm{HD}$ & -0.08 & .932 \\
\hline & S>Categorization & 1.52 & $<.001$ \\
\hline & $\mathrm{N}>$ Categorization & 0.22 & .114 \\
\hline \multirow[t]{4}{*}{ AngG } & Categorization $>0$ & 0.24 & .165 \\
\hline & $\mathrm{LD}>\mathrm{HD}$ & -0.30 & .575 \\
\hline & S>Categorization & 0.57 & $<.001$ \\
\hline & $\mathrm{N}>$ Categorization & -0.34 & .086 \\
\hline
\end{tabular}

Table 2. Mixed-effect linear regression results for multiple demand fROIs. P-values were FDRcorrected for the number of fROIs. Significant p-values are highlighted in bold. $H$ - hard working memory task, E - easy working memory task, LD - low-dimensional categorization, HD - highdimensional categorization.

\begin{tabular}{|c|c|c|c|c|c|}
\hline Hemisphere & fROI \# & fROI name & Regression Term & Beta & $p$-value \\
\hline \multirow[t]{6}{*}{$\mathrm{L}$} & 1 & postParietal & Categorization $>0$ & 1.44 & $<.001$ \\
\hline & & & $\mathrm{LD}>\mathrm{HD}$ & 0.28 & .902 \\
\hline & & & Hard WM>Easy WM & 1.37 & $<.001$ \\
\hline & & & WM>Categorization & 2.69 & $<.001$ \\
\hline & & & Nonwords>Categorization & -0.90 & $<.001$ \\
\hline & & & Sentences>Categorization & -1.36 & $<.001$ \\
\hline \multirow[t]{6}{*}{$\mathrm{L}$} & 2 & midParietal & Categorization $>0$ & 0.98 & .004 \\
\hline & & & $\mathrm{LD}>\mathrm{HD}$ & 0.30 & .902 \\
\hline & & & Hard WM > Easy WM & 1.40 & $<.001$ \\
\hline & & & WM>Categorization & 2.32 & $<.001$ \\
\hline & & & Nonwords>Categorization & -0.07 & .913 \\
\hline & & & Sentences>Categorization & -0.65 & .034 \\
\hline \multirow[t]{6}{*}{$\mathrm{L}$} & 3 & antParietal & Categorization $>0$ & 0.88 & .003 \\
\hline & & & $\mathrm{LD}>\mathrm{HD}$ & 0.47 & .902 \\
\hline & & & Hard WM>Easy WM & 1.14 & $<.001$ \\
\hline & & & WM>Categorization & 2.10 & $<.001$ \\
\hline & & & Nonwords>Categorization & -0.17 & .567 \\
\hline & & & Sentences $>$ Categorization & -0.71 & .004 \\
\hline \multirow[t]{3}{*}{$\mathrm{L}$} & 4 & supFrontal & Categorization $>0$ & 0.77 & .007 \\
\hline & & & $\mathrm{LD}>\mathrm{HD}$ & 0.23 & .902 \\
\hline & & & Hard WM>Easy WM & 0.97 & .002 \\
\hline
\end{tabular}




\begin{tabular}{|c|c|c|c|c|c|}
\hline & & & WM>Categorization & 2.16 & $<.001$ \\
\hline & & & Nonwords $>$ Categorization & -0.28 & .355 \\
\hline & & & Sentences $>$ Categorization & -0.38 & .139 \\
\hline \multirow[t]{6}{*}{$\mathrm{L}$} & 5 & precentral_A & Categorization $>0$ & 2.23 & $<.001$ \\
\hline & & & $\mathrm{LD}>\mathrm{HD}$ & 0.37 & .902 \\
\hline & & & Hard WM>Easy WM & 1.34 & $<.001$ \\
\hline & & & WM>Categorization & 0.98 & $<.001$ \\
\hline & & & Nonwords $>$ Categorization & -0.56 & .058 \\
\hline & & & Sentences $>$ Categorization & -0.97 & $<.001$ \\
\hline \multirow[t]{6}{*}{$\mathrm{L}$} & 6 & precentral_B & Categorization $>0$ & 1.42 & $<.001$ \\
\hline & & & $\mathrm{LD}>\mathrm{HD}$ & 0.17 & .902 \\
\hline & & & Hard WM>Easy WM & 1.12 & $<.001$ \\
\hline & & & WM $>$ Categorization & 0.71 & .001 \\
\hline & & & Nonwords $>$ Categorization & -0.66 & .030 \\
\hline & & & Sentences $>$ Categorization & -0.77 & .004 \\
\hline \multirow[t]{6}{*}{$\mathrm{L}$} & 7 & midFrontal & Categorization $>0$ & 1.29 & $<.001$ \\
\hline & & & $\mathrm{LD}>\mathrm{HD}$ & 0.18 & .902 \\
\hline & & & Hard WM>Easy WM & 0.94 & $<.001$ \\
\hline & & & $\mathrm{WM}>$ Categorization & 0.25 & .178 \\
\hline & & & Nonwords $>$ Categorization & -0.86 & .002 \\
\hline & & & Sentences $>$ Categorization & -1.25 & $<.001$ \\
\hline \multirow[t]{6}{*}{$\mathrm{L}$} & 8 & midFrontalOrb & Categorization $>0$ & 1.15 & .006 \\
\hline & & & $\mathrm{LD}>\mathrm{HD}$ & 0.13 & .902 \\
\hline & & & Hard WM>Easy WM & 1.29 & $<.001$ \\
\hline & & & WM>Categorization & 0.65 & .003 \\
\hline & & & Nonwords $>$ Categorization & -0.62 & .034 \\
\hline & & & Sentences $>$ Categorization & -1.08 & $<.001$ \\
\hline \multirow[t]{6}{*}{$\mathrm{L}$} & 9 & insula & Categorization $>0$ & 0.81 & $<.001$ \\
\hline & & & $\mathrm{LD}>\mathrm{HD}$ & -0.01 & .953 \\
\hline & & & Hard WM>Easy WM & 0.72 & $<.001$ \\
\hline & & & WM $>$ Categorization & 0.49 & $<.001$ \\
\hline & & & Nonwords $>$ Categorization & -0.38 & .008 \\
\hline & & & Sentences $>$ Categorization & -0.49 & $<.001$ \\
\hline \multirow[t]{6}{*}{$\mathrm{L}$} & 10 & medialFrontal & Categorization $>0$ & 0.95 & $<.001$ \\
\hline & & & $\mathrm{LD}>\mathrm{HD}$ & -0.01 & .953 \\
\hline & & & Hard WM>Easy WM & 0.79 & $<.001$ \\
\hline & & & WM>Categorization & 0.59 & $<.001$ \\
\hline & & & Nonwords $>$ Categorization & -0.40 & .033 \\
\hline & & & Sentences $>$ Categorization & -0.56 & .001 \\
\hline \multirow[t]{6}{*}{$\mathrm{R}$} & 1 & postParietal & Categorization $>0$ & 1.15 & $<.001$ \\
\hline & & & $\mathrm{LD}>\mathrm{HD}$ & 0.26 & .902 \\
\hline & & & Hard WM>Easy WM & 1.68 & $<.001$ \\
\hline & & & WM $>$ Categorization & 3.18 & $<.001$ \\
\hline & & & Nonwords $>$ Categorization & -0.84 & .009 \\
\hline & & & Sentences $>$ Categorization & -1.14 & $<.001$ \\
\hline \multirow[t]{6}{*}{$\mathrm{R}$} & 2 & midParietal & Categorization $>0$ & 0.74 & .004 \\
\hline & & & $\mathrm{LD}>\mathrm{HD}$ & 0.31 & .902 \\
\hline & & & Hard WM>Easy WM & 1.72 & $<.001$ \\
\hline & & & WM>Categorization & 2.09 & $<.001$ \\
\hline & & & Nonwords $>$ Categorization & 0.05 & .913 \\
\hline & & & Sentences $>$ Categorization & -0.43 & .148 \\
\hline \multirow[t]{6}{*}{$\mathrm{R}$} & 3 & antParietal & Categorization $>0$ & 0.33 & .040 \\
\hline & & & $\mathrm{LD}>\mathrm{HD}$ & 0.35 & .902 \\
\hline & & & Hard WM>Easy WM & 1.23 & $<.001$ \\
\hline & & & WM $>$ Categorization & 1.98 & $<.001$ \\
\hline & & & Nonwords $>$ Categorization & 0.00 & .989 \\
\hline & & & Sentences $>$ Categorization & -0.27 & .273 \\
\hline \multirow[t]{3}{*}{$\mathrm{R}$} & 4 & supFrontal & Categorization $>0$ & 0.70 & .013 \\
\hline & & & $\mathrm{LD}>\mathrm{HD}$ & 0.14 & .902 \\
\hline & & & Hard WM>Easy WM & 1.55 & $<.001$ \\
\hline
\end{tabular}




\begin{tabular}{|c|c|c|c|c|c|}
\hline & & & WM>Categorization & 2.77 & $<.001$ \\
\hline & & & Nonwords $>$ Categorization & -0.06 & .913 \\
\hline & & & Sentences $>$ Categorization & -0.20 & .526 \\
\hline \multirow[t]{6}{*}{$\mathrm{R}$} & 5 & precentral_A & Categorization $>0$ & 1.48 & $<.001$ \\
\hline & & & $\mathrm{LD}>\mathrm{HD}$ & 0.19 & .902 \\
\hline & & & Hard WM>Easy WM & 1.40 & $<.001$ \\
\hline & & & WM>Categorization & 1.22 & $<.001$ \\
\hline & & & Nonwords>Categorization & -0.35 & .353 \\
\hline & & & Sentences $>$ Categorization & -0.68 & .032 \\
\hline \multirow[t]{6}{*}{$\mathrm{R}$} & 6 & precentral_B & Categorization $>0$ & 1.73 & $<.001$ \\
\hline & & & $\mathrm{LD}>\mathrm{HD}$ & 0.29 & .902 \\
\hline & & & Hard WM>Easy WM & 1.65 & $<.001$ \\
\hline & & & WM $>$ Categorization & 1.21 & $<.001$ \\
\hline & & & Nonwords>Categorization & -0.72 & .067 \\
\hline & & & Sentences $>$ Categorization & -1.08 & .004 \\
\hline \multirow[t]{6}{*}{$\mathrm{R}$} & 7 & midFrontal & Categorization $>0$ & 0.91 & .009 \\
\hline & & & $\mathrm{LD}>\mathrm{HD}$ & 0.17 & .902 \\
\hline & & & Hard WM>Easy WM & 1.71 & $<.001$ \\
\hline & & & WM $>$ Categorization & 1.08 & $<.001$ \\
\hline & & & Nonwords>Categorization & -0.26 & .485 \\
\hline & & & Sentences $>$ Categorization & -0.64 & .036 \\
\hline \multirow[t]{6}{*}{$\mathrm{R}$} & 8 & midFrontalOrb & Categorization $>0$ & 0.89 & .003 \\
\hline & & & $\mathrm{LD}>\mathrm{HD}$ & 0.05 & .953 \\
\hline & & & Hard WM>Easy WM & 1.89 & $<.001$ \\
\hline & & & WM>Categorization & 0.96 & $<.001$ \\
\hline & & & Nonwords $>$ Categorization & -0.39 & .306 \\
\hline & & & Sentences $>$ Categorization & -0.86 & .007 \\
\hline \multirow[t]{6}{*}{$\mathrm{R}$} & 9 & insula & Categorization $>0$ & 0.72 & .001 \\
\hline & & & $\mathrm{LD}>\mathrm{HD}$ & -0.04 & .902 \\
\hline & & & Hard WM>Easy WM & 0.85 & $<.001$ \\
\hline & & & WM>Categorization & 0.42 & $<.001$ \\
\hline & & & Nonwords $>$ Categorization & -0.34 & .013 \\
\hline & & & Sentences $>$ Categorization & -0.46 & $<.001$ \\
\hline \multirow[t]{6}{*}{$\mathrm{R}$} & 10 & medialFrontal & Categorization $>0$ & 0.84 & $<.001$ \\
\hline & & & $\mathrm{LD}>\mathrm{HD}$ & 0.06 & .902 \\
\hline & & & Hard WM>Easy WM & 1.23 & $<.001$ \\
\hline & & & WM>Categorization & 0.63 & $<.001$ \\
\hline & & & Nonwords $>$ Categorization & -0.35 & .073 \\
\hline & & & Sentences $>$ Categorization & -0.60 & .002 \\
\hline
\end{tabular}

